\title{
Role of Benzylic Deprotonation in Ni-Catalyzed Benzylic Dehydrogenation
}

\author{
Pengpeng Zhang, Rachel L. Cantrell, and Timothy R. Newhouse* \\ Department of Chemistry, Yale University \\ 225 Prospect St., New Haven, Connecticut 06520-8107 \\ *E-mail: timothy.newhouse@yale.edu
}

\section{Table of Contents}

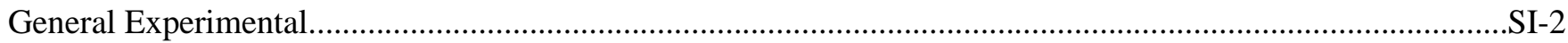

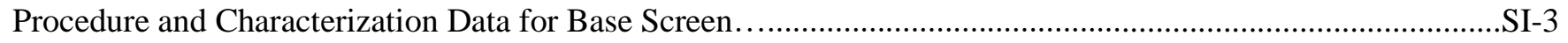

Procedure and Characterization Data for Temperature-Dependent Studies.....................................................SI-7

Procedure and Characterization Data for Time-Dependent Studies ..........................................................SI-12

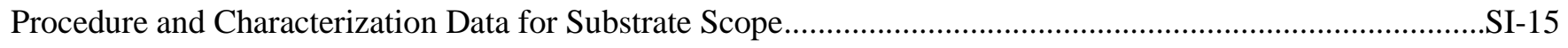

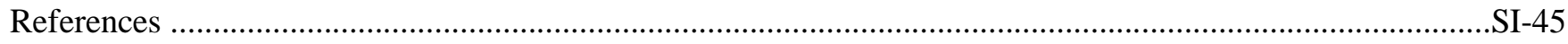




\section{$\underline{\text { General Experimental }}$}

General Experimental Procedures: All reactions were carried out under an inert nitrogen atmosphere with dry solvents under anhydrous conditions unless otherwise stated. All reactions were capped with a rubber septum, or Teflon-coated silicon microwave cap unless otherwise stated. Stainless steel cannula or syringe was used to transfer solvent, and air- and moisture sensitive liquid reagents. Reactions were monitored by thin-layer chromatography (TLC) carried out on $0.25 \mathrm{~mm}$ Merck silica gel plates (60F-254) using UV light as the visualizing agent and potassium permanganate, an acidic solution of $p$-anisaldehyde, phosphomolybdic acid, or $\mathrm{I}_{2}$ on $\mathrm{SiO}_{2}$ as developing agents. Flash column chromatography employed SiliaFlash ${ }^{\circledR}$ P60 (40-60 $\mu \mathrm{m}, 230-400$ mesh) silica gel purchased from SiliCycle, Inc.

Materials: All reaction solvents were purified using a Seca solvent purification system by Glass contour. TMEDA, $\mathrm{Et}_{3} \mathrm{~N}$ were distilled over $\mathrm{CaH}_{2}$. $\mathrm{Zn}(\mathrm{TMP})_{2}\left(0.5 \mathrm{M}\right.$ in toluene), $\mathrm{PMe}_{3}(1.0 \mathrm{M}$ in THF), TBAI and $n$-BuLi (2.5 M in hexanes) were purchased from Sigma-Aldrich. $\mathrm{NiBr}_{2}$ (dme) was purchased from Strem. All other reagents were used as received without further purification, unless otherwise stated.

Instrumentation: All new compounds were characterized by means of ${ }^{1} \mathrm{H}$ NMR, ${ }^{13} \mathrm{C}$ NMR, ${ }^{19} \mathrm{~F}$ NMR, FT-IR (thin film), and HR-MS. Copies of the ${ }^{1} \mathrm{H}$ - and ${ }^{13} \mathrm{C}-\mathrm{NMR}$ spectra can be found at the end of each experimental procedure. NMR spectra were recorded using a Varian $400 \mathrm{MHz}$ NMR spectrometer, Varian $500 \mathrm{MHz}$ NMR spectrometer, or a Varian $600 \mathrm{MHz}$ NMR spectrometer. All ${ }^{1} \mathrm{H}-\mathrm{NMR}$ data are reported in $\delta$ units, parts per million (ppm), and were calibrated relative to the signals for residual chloroform (7.26 $\mathrm{ppm})$ in deuterochloroform $\left(\mathrm{CDCl}_{3}\right) .{ }^{1} \mathrm{H} \mathrm{NMR}$ for starting materials and after deuteration were acquired with the same protocol (instrument, D1, number of scans, etc.) in order to ensure cancellation of error due to variable T1 relaxation times. All ${ }^{13} \mathrm{C}-\mathrm{NMR}$ data are reported in ppm relative to $\mathrm{CDCl}_{3}(77.16 \mathrm{ppm})$ or Acetone- $d_{6}(206.26 \mathrm{ppm})$ and were obtained with ${ }^{1} \mathrm{H}$ decoupling unless otherwise stated. The following abbreviations or combinations thereof were used to explain the multiplicities: $\mathrm{s}=$ singlet, $\mathrm{d}=$ doublet, $\mathrm{t}=$ triplet, $\mathrm{q}=$ quartet, $\mathrm{abq}=\mathrm{ab}$ quartet, $\mathrm{br}=$ broad, $\mathrm{m}=$ multiplet, and $\mathrm{a}=$ apparent. All IR spectra were taken on an FT-IR/Raman Thermo Nicolet 6700. High resolution mass spectra (HR-MS) were recorded on a Waters Xevo Qtof mass spectrometer using ESI-TOF (electrospray ionization-time of flight). Gas chromatography mass spectra (GC-MS) were recorded on an Agilent Technologies 6890N Network Gas Chromatograph System with an Agilent Technologies 5973N Mass Selective Detector. Optical rotation data was obtained using a Perkin-Elmer 341 polarimeter. 


\section{Procedure and Characterization Data for Base Screen}

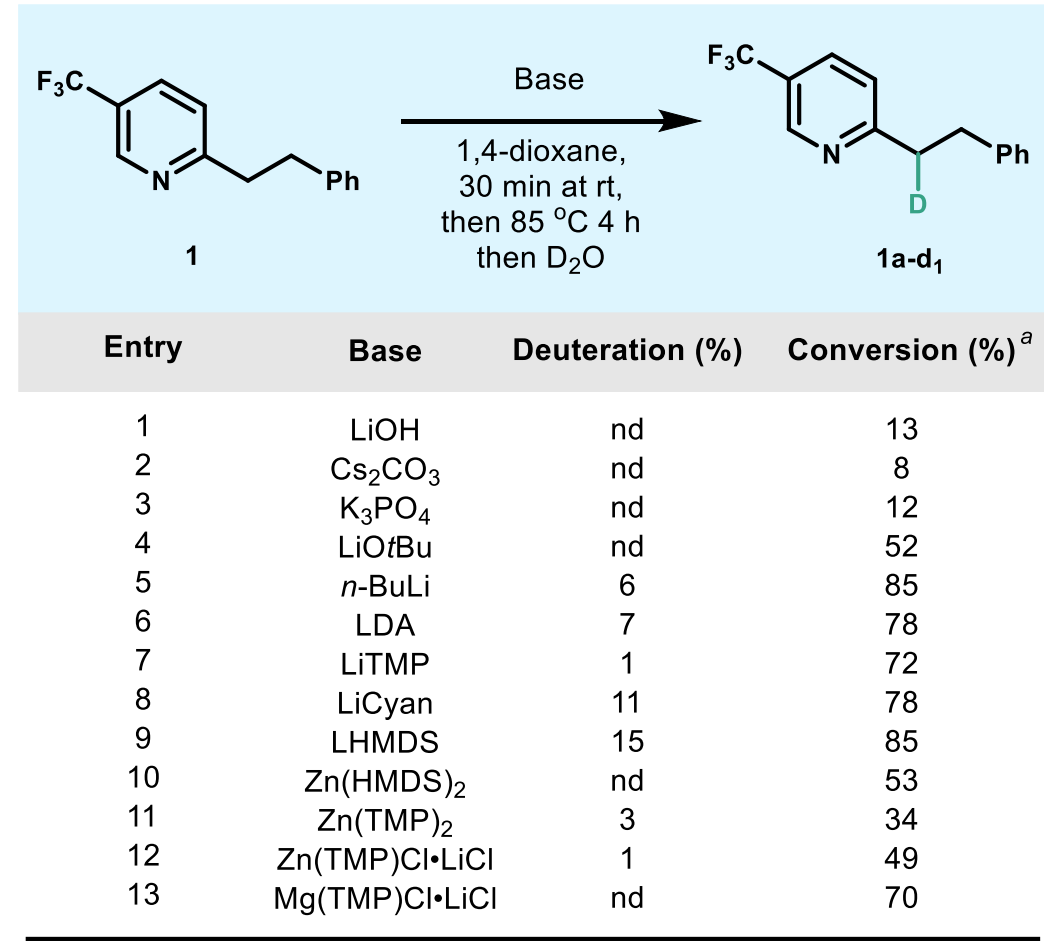

[a] Yield of deuterated and conversion were determined by ${ }^{1} \mathrm{H}$ NMR analysis using 1,3,5trimethoxybenzene as an internal standard. [b] Bases in entry 12 were made via the procedure by Zhao et. al. ${ }^{1}$

\section{General Procedure A (entry 1-3):}

To a flame-dried microwave vial equipped with a magnetic stir bar was added solid bases (0.075mmol, 1.5 equiv). The reaction vessel was evacuated and backfilled with $\mathrm{N}_{2}$ (this process was repeated three times). To the reaction vessel was added a solution of starting material $(0.05 \mathrm{mmol}, 1$ equiv.) in anhydrous 1,4-dioxane $(0.1 \mathrm{M}, 0.5 \mathrm{~mL})$. The reaction was allowed to stir at room temperature for 30 minutes before being placed in an $85^{\circ} \mathrm{C}$ oil bath, where it was stirred for 4 hours.

Immediately after removal from heat, the reaction was quenched with $\mathrm{D}_{2} \mathrm{O}(0.5 \mathrm{~mL})$ and stirred for 5 minutes to cool down to room temperature. A solution of internal standard 1,3,5-trimethoxybenzene in EtOAc was added before extraction with EtOAc $(4 \times 1.5 \mathrm{~mL})$, and the combined organic extracts were dried with $\mathrm{Na}_{2} \mathrm{SO}_{4}$ and filtered through a short silica plug and washed with EtOAc $(5 \mathrm{~mL})$ and concentrated under reduced pressure by rotary evaporation. Then $0.2 \mathrm{~mL}$ of $\mathrm{CDCl}_{3}$ was added and the excess solvent was removed under vacuum. Finally, the deuteration and degradation percentage was determined by crude ${ }^{1} \mathrm{H}$ NMR based on the internal standard. 


\section{General Procedure B (entry 4-13):}

A flame-dried microwave vial equipped with a magnetic stir bar was evacuated and backfilled with $\mathrm{N}_{2}$ (this process was repeated three times). To the reaction vessel was added a solution of starting material (0.05 mmol, 1 equiv.) in anhydrous 1,4 -dioxane $(0.1 \mathrm{M}, 1 \mathrm{~mL})$, followed by the solution of commercially available or fresh-made base $(0.075 \mathrm{mmol}, 1.5$ equiv). The reaction was stirred at room temperature for 30 mins and then moved to an $85^{\circ} \mathrm{C}$ oil bath for 4 hours.

Immediately after removal from heat, the reaction was quenched with $\mathrm{D}_{2} \mathrm{O}(0.5 \mathrm{~mL})$ and stirred for 5 minutes to cool down to room temperature. A solution of internal standard 1,3,5-trimethoxybenzene in EtOAc was added before extraction with EtOAc $(4 \times 1.5 \mathrm{~mL})$, and the combined organic extracts were dried with $\mathrm{Na}_{2} \mathrm{SO}_{4}$ and filtered through a short silica plug and washed with EtOAc $(5 \mathrm{~mL})$ and concentrated under reduced pressure by rotary evaporation. Then $0.2 \mathrm{~mL}$ of $\mathrm{CDCl}_{3}$ was added and the excess solvent was removed under vacuum. Finally, the deuteration and degradation percentage was determined by crude ${ }^{1} \mathrm{H}$ NMR based on the internal standard.

Note: 2 equiv. of internal standard was added in entries 1-3, and 11.

\section{2-phenethyl-5-(trifluoromethyl)pyridine (1)}

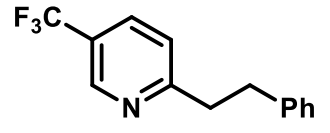

Compound 1 was prepared according to known procedure, ${ }^{2}$ and the characterization data match those previously reported in the literature.

${ }^{1} \mathbf{H}$ NMR $\left(400 \mathrm{MHz}_{\mathrm{CDCl}}\right): \delta 8.85(\mathrm{~s}, 1 \mathrm{H}), 7.79(\mathrm{dd}, J=8.2,2.4 \mathrm{~Hz}, 1 \mathrm{H}), 7.32-7.27(\mathrm{~m}, 2 \mathrm{H}), 7.24-$ $7.16(\mathrm{~m}, 4 \mathrm{H}), 3.21-3.16(\mathrm{~m}, 2 \mathrm{H}), 3.13-3.07(\mathrm{~m}, 2 \mathrm{H})$

${ }^{13} \mathrm{C}$ NMR (101 MHz, $\left.\mathrm{CDCl}_{3}\right): \delta 165.4,146.4\left(\mathrm{q}, J_{C-F}=4.1 \mathrm{~Hz}\right), 141.0,133.4\left(\mathrm{q}, J_{C-F}=3.5 \mathrm{~Hz}\right), 128.6$, $128.5,126.6,124.4\left(\mathrm{q}, J_{C-F}=33.0 \mathrm{~Hz}\right), 123.9\left(\mathrm{q}, J_{C-F}=272.1 \mathrm{~Hz}\right), 122.8,40.2,35.7$

${ }^{19}$ F NMR (376 MHz, $\left.\mathrm{CDCl}_{3}\right): \delta-62.2(\mathrm{~s})$

ESI-HRMS (m/z): $[\mathrm{M}+\mathrm{H}]^{+}$calc'd for $\mathrm{C}_{14} \mathrm{H}_{13} \mathrm{~F}_{3} \mathrm{~N}^{+}:$: 252.0995; found: 252.1000 

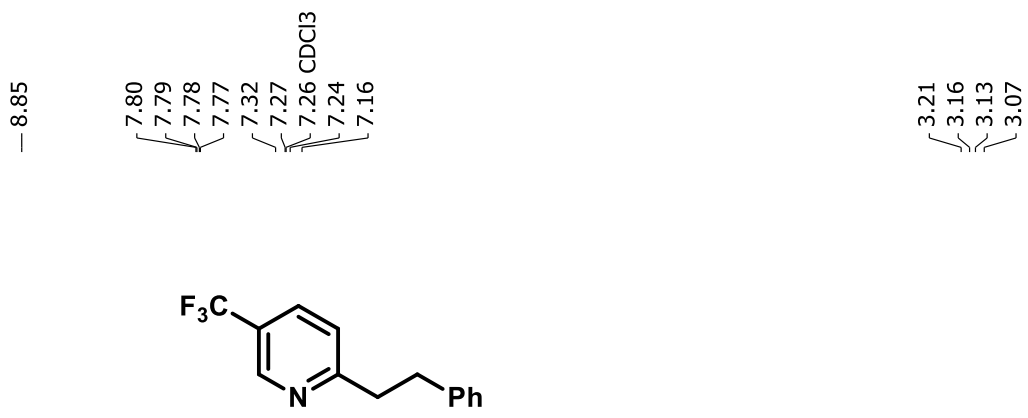

1

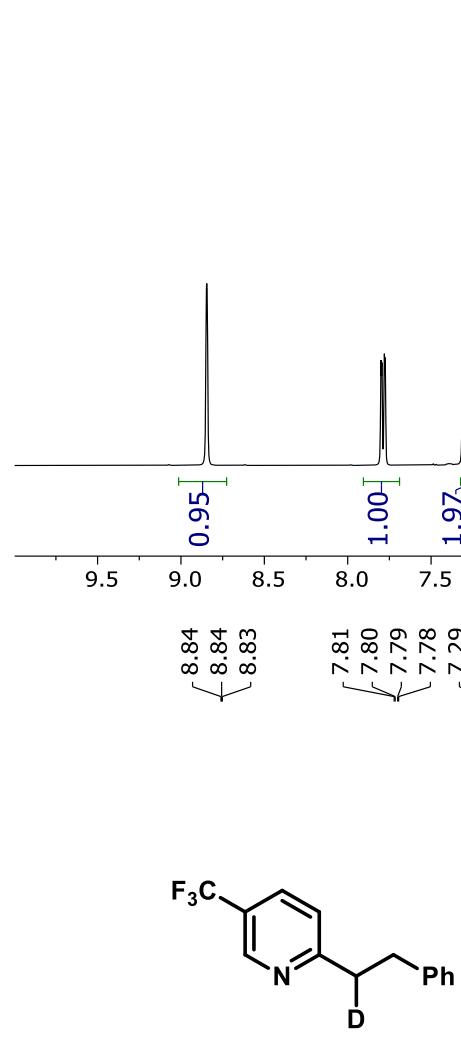

Entry $2\left(\mathrm{Cs}_{2} \mathrm{CO}_{3}\right)$

Degradation:

$1-0.97 /(2.11 / 2)=8 \%$

(with 2 equiv. internal standard)

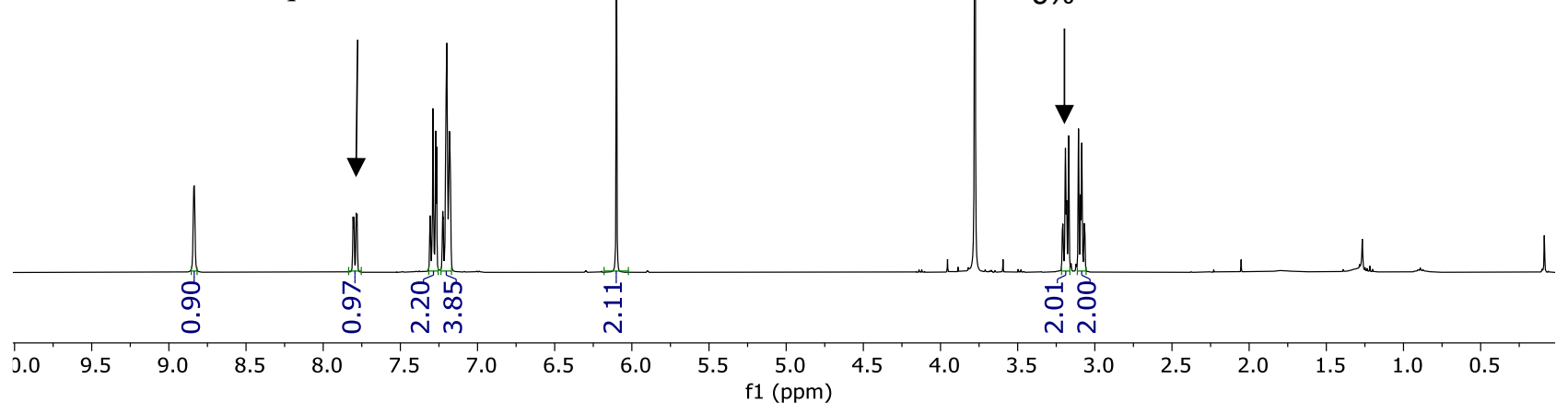

SI-5 


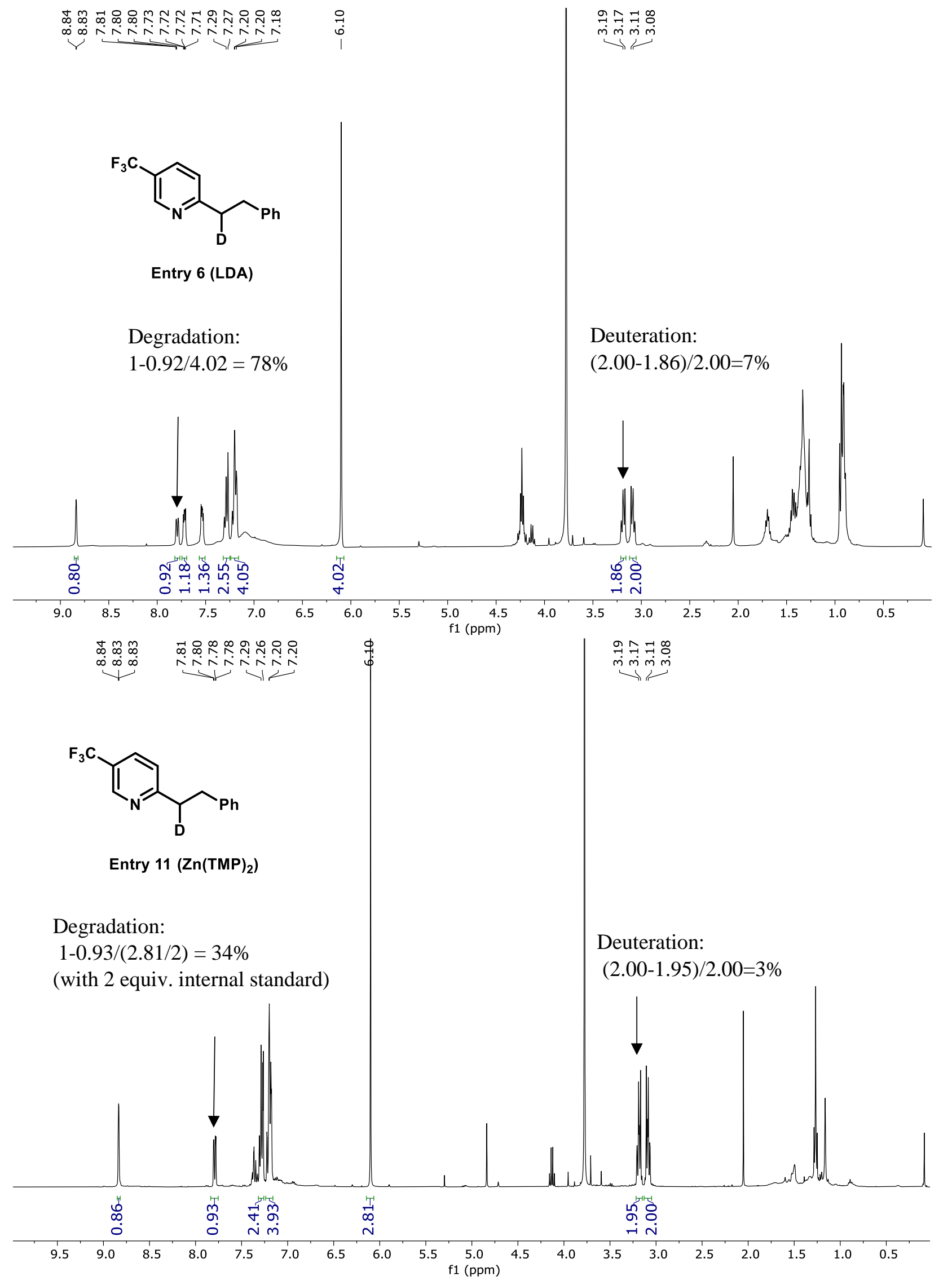




\section{Procedure and Characterization Data for Temperature-Dependent Studies}

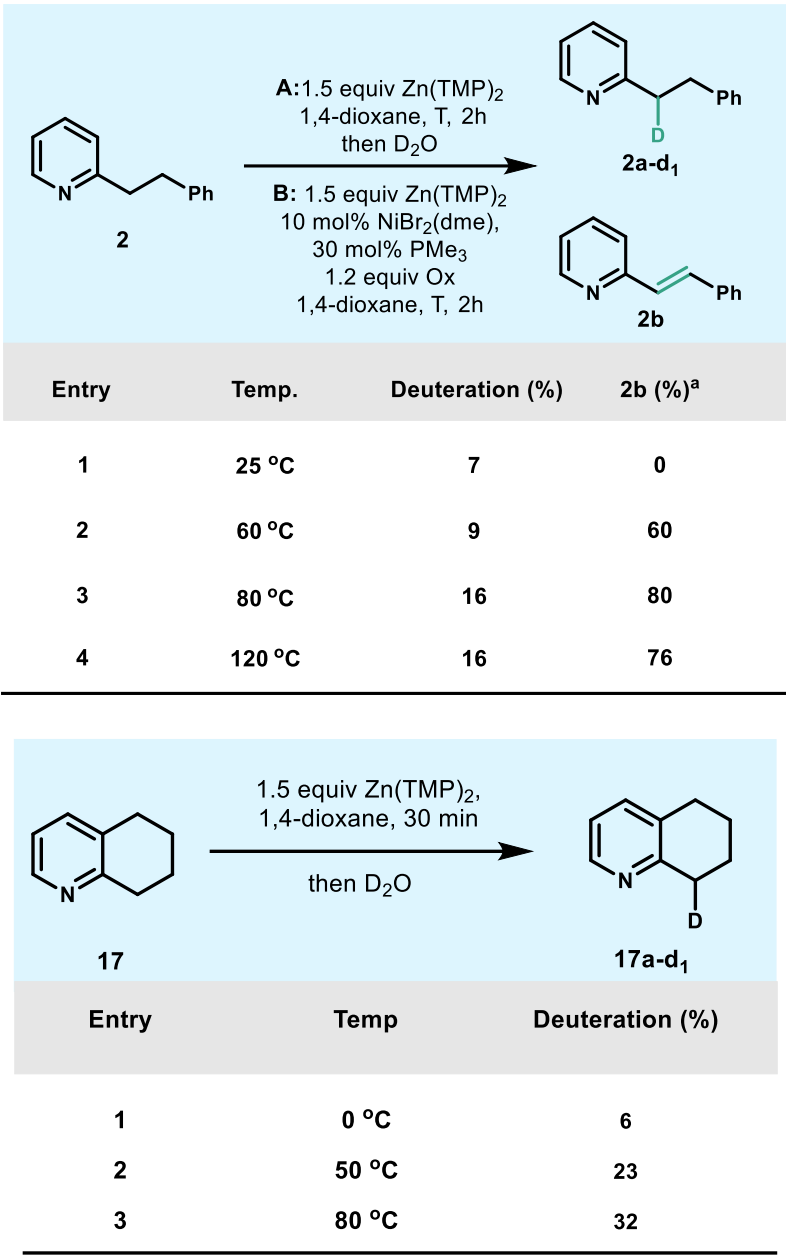

[a] Yield of dehydrogenation was determined by ${ }^{1} \mathrm{H}$ NMR analysis using 1,3,5trimethoxybenzene as an internal standard. Ox $=2$-bromo-5-methylthiophene

\section{General Procedure C: (condition A)}

A flame-dried microwave vial equipped with a magnetic stir bar was evacuated and backfilled with $\mathrm{N}_{2}$ (this process was repeated three times). To the reaction vessel was added a solution of starting material 2 ( $0.1 \mathrm{mmol}, 1$ equiv.) in anhydrous 1,4-dioxane $(0.1 \mathrm{M}, 1 \mathrm{~mL})$, followed by the solution of commercial $\mathrm{Zn}(\mathrm{TMP})_{2}(0.3 \mathrm{~mL}, 0.5 \mathrm{M}$ in toulene, $0.15 \mathrm{mmol}, 1.5$ equiv $)$. The reaction was stirred at different temperature for $2 \mathrm{~h}$ as the table shows.

Immediately after removal from heat, the reaction was quenched with $\mathrm{D}_{2} \mathrm{O}(0.5 \mathrm{~mL})$ and stirred for 5 minutes to cool down to room temperature. A solution of internal standard 1,3,5-trimethoxybenzene in EtOAc was added ( $0.33 \mathrm{mmol}$ unless otherwise stated) before extraction with EtOAc $(4 \times 1.5 \mathrm{~mL})$, and the combined organic extracts were dried with $\mathrm{Na}_{2} \mathrm{SO}_{4}$ and filtered through a short silica plug and washed with EtOAc $(5 \mathrm{~mL})$ and concentrated under reduced pressure by rotary evaporation. Then $0.2 \mathrm{~mL}$ of $\mathrm{CDCl}_{3}$ 
was added and the excess solvent was removed under vacuum. Finally, the deuteration and degradation percentage was determined by crude ${ }^{1} \mathrm{H}$ NMR based on the internal standard.

\section{General Procedure D: $(\text { condition B })^{2}$}

To a flame-dried microwave vial equipped with a magnetic stir bar was added starting material 2 ( $0.20 \mathrm{mmol}, 1.0$ equiv). The reaction vessel was sealed, evacuated and backfilled with $\mathrm{N}_{2}$ (this process was repeated three times). To the reaction vessel was added anhydrous 1,4-dioxane (1.5 $\mathrm{mL})$ and commercial $\mathrm{Zn}(\mathrm{TMP})_{2}(0.48 \mathrm{~mL}, 0.5 \mathrm{M}$ in toluene, $0.3 \mathrm{mmol}, 1.5$ equiv $)$ at room temperature. Then to this mixture was added $0.5 \mathrm{~mL}$ of a stock solution containing $\mathrm{NiBr}_{2}(\mathrm{dme})(6.2 \mathrm{mg}, 0.02 \mathrm{mmol}, 10 \mathrm{~mol}$ $\%), \mathrm{PMe}_{3}(0.06 \mathrm{~mL}, 0.06 \mathrm{mmol}, 30 \mathrm{~mol} \%)$, and 2-bromo-5-methylthiophene (27.4 uL, $0.24 \mathrm{mmol}, 1.2$ equiv) in 1,4-dioxane. The reaction vessel was placed into a pre-heated oil bath for $2 \mathrm{~h}$ (at different temperature as table shows).

The reaction mixture was cooled to ambient temperature and quenched by the addition of sat. aq. $\mathrm{NH}_{4} \mathrm{Cl}(5 \mathrm{~mL})$. The reaction mixture was diluted with EtOAc $(5 \mathrm{~mL})$ and the organic phase was separated. The aqueous phase was extracted with EtOAc $(3 \times 5 \mathrm{~mL})$ and the combined organic extracts were washed with brine $(5 \mathrm{~mL})$, dried over anhydrous $\mathrm{Na}_{2} \mathrm{SO}_{4}$, filtered, and concentrated under reduced pressure by rotary evaporation. Yield was determined by crude ${ }^{1} \mathrm{H}$ NMR based on the internal standard. 


\section{2-phenethylpyridine (2)}<smiles>c1ccc(CCc2cccnc2)nc1</smiles>

Compound 2 was prepared according to known procedure, ${ }^{2}$ and the characterization data match those previously reported in the literature.

${ }^{1} \mathbf{H}$ NMR $\left(400 \mathrm{MHz}, \mathrm{CDCl}_{3}\right) \delta 8.57(\mathrm{~d}, J=4.1 \mathrm{~Hz}, 1 \mathrm{H}), 7.55(\mathrm{td}, J=7.7,1.8 \mathrm{~Hz}, 1 \mathrm{H}), 7.31-7.24(\mathrm{~m}$, $2 \mathrm{H}), 7.23-7.18(\mathrm{~m}, 3 \mathrm{H}), 7.06-7.13(\mathrm{~m}, 2 \mathrm{H}), 3.13-3.04(\mathrm{~m}, 4 \mathrm{H})$.

The deuteration is determined by the compare the integration change of benzylic protons at $3.13-3.04$ (m, 4H) with compound 2 by setting integration of $7.55 \mathrm{ppm}$ as 1.00 .

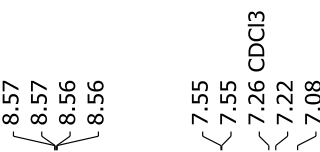

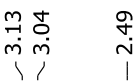

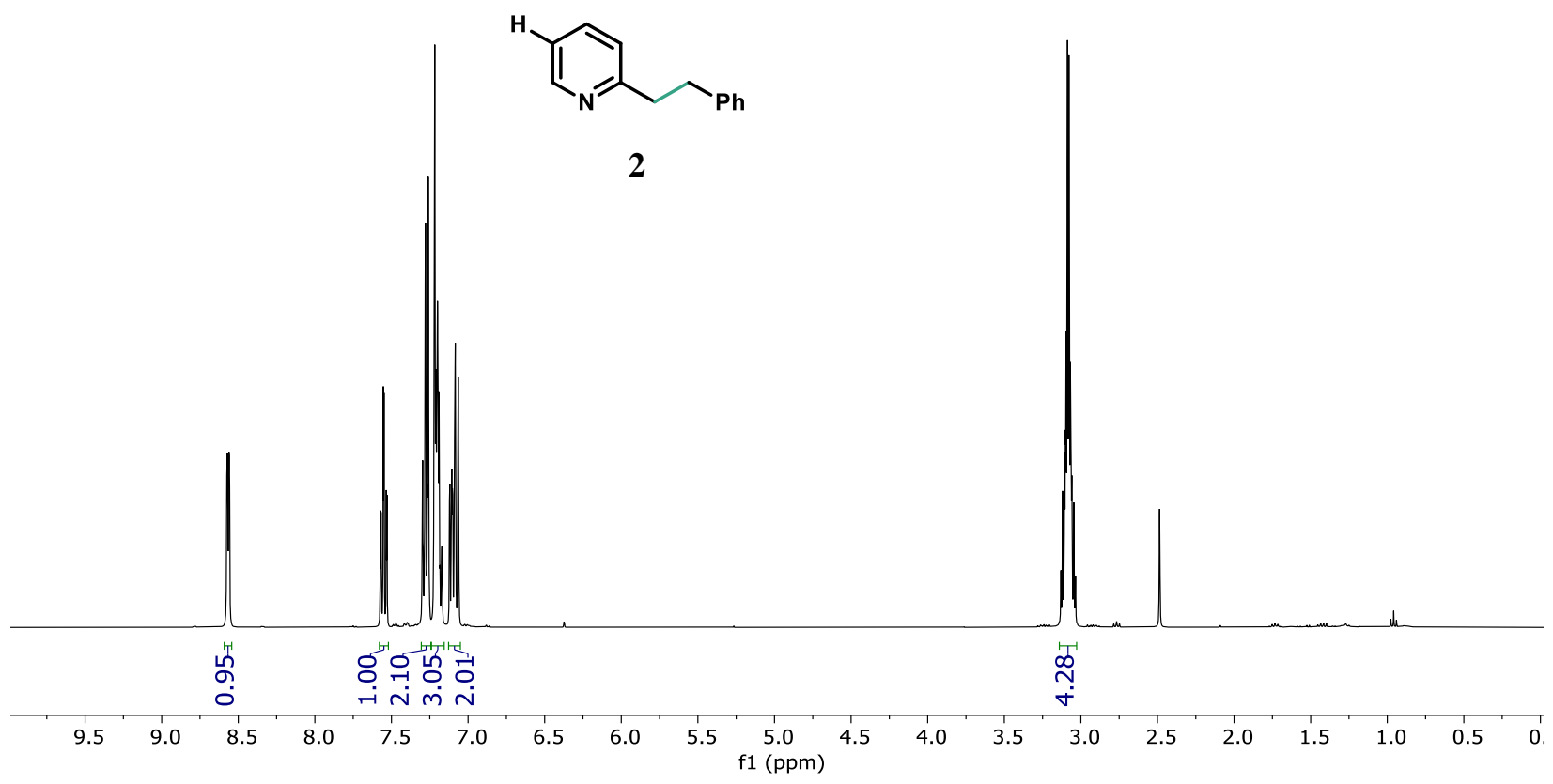




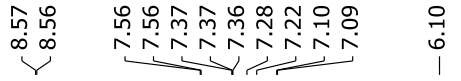

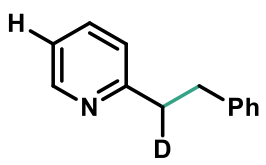

2a (Entry 1, $\left.25^{\circ} \mathrm{C}\right)$ $\stackrel{\circ}{\circ} \stackrel{\infty}{m}$

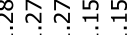

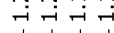

Deuteration:

$(4.28-4.13) /(4.28 / 2)=7 \%$

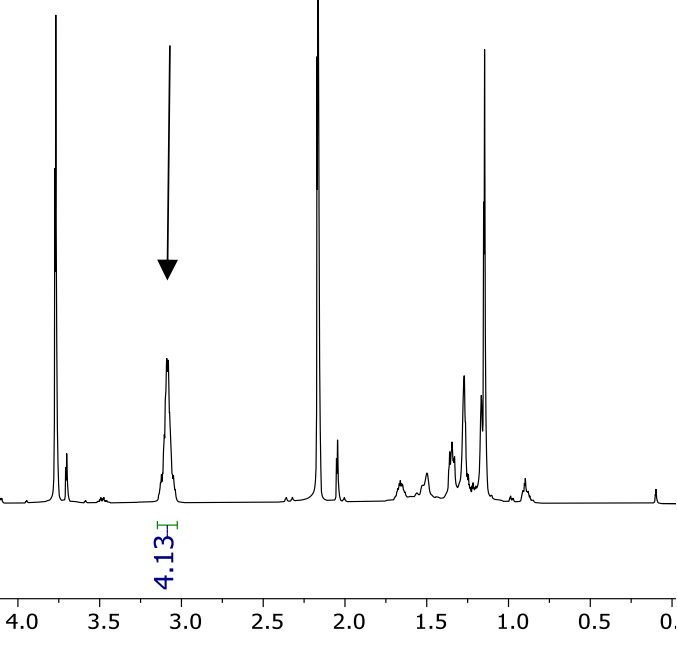

पับ

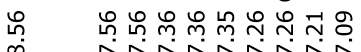

i nininin

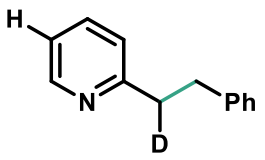

Deuteration:

2a (Entry 2: $\left.60{ }^{\circ} \mathrm{C}\right)$
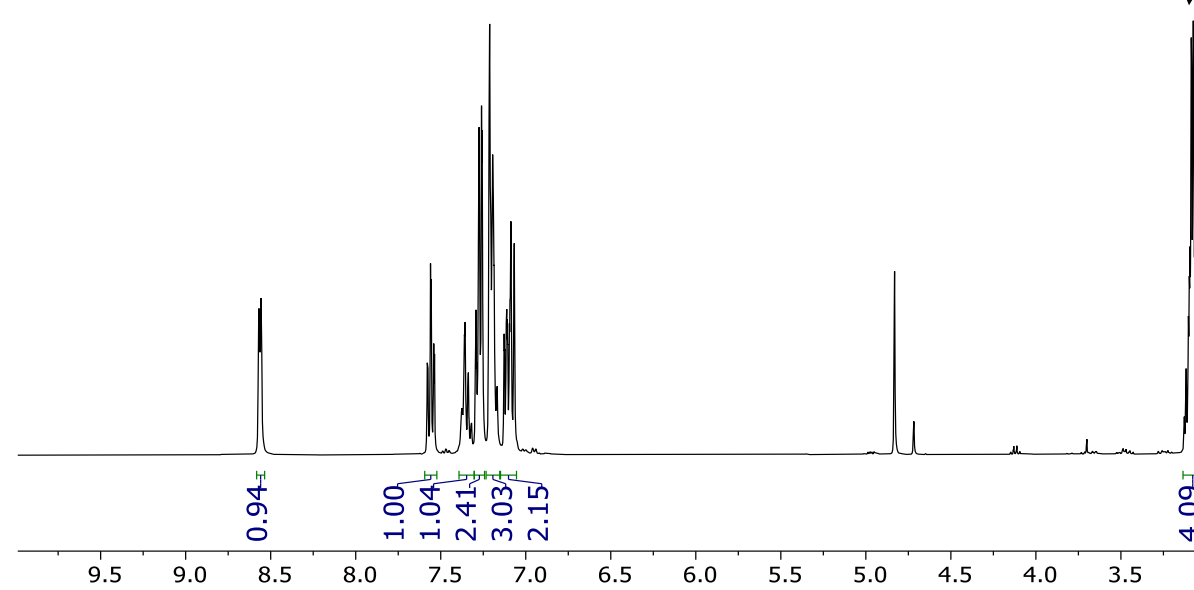

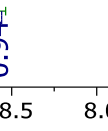

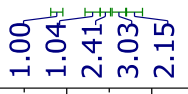

$\begin{array}{lll}7.5 & 7.0 & 6.5\end{array}$

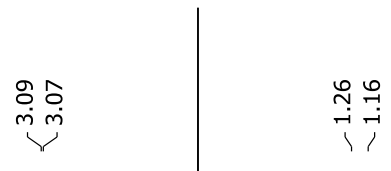

$(4.28-4.09) /(4.28 / 2)=9 \%$

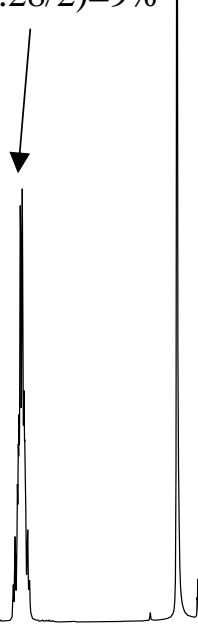

宁

i

\section{,}

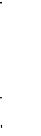


H<smiles>OC(Cc1ccccc1)c1ccccn1</smiles>

2a (Entry 3: $\left.80^{\circ} \mathrm{C}\right)$
Deuteration:

$(4.28-3.93) /(4.28 / 2)=16 \%$

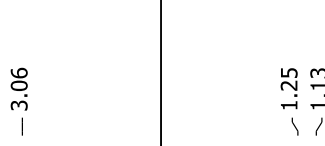
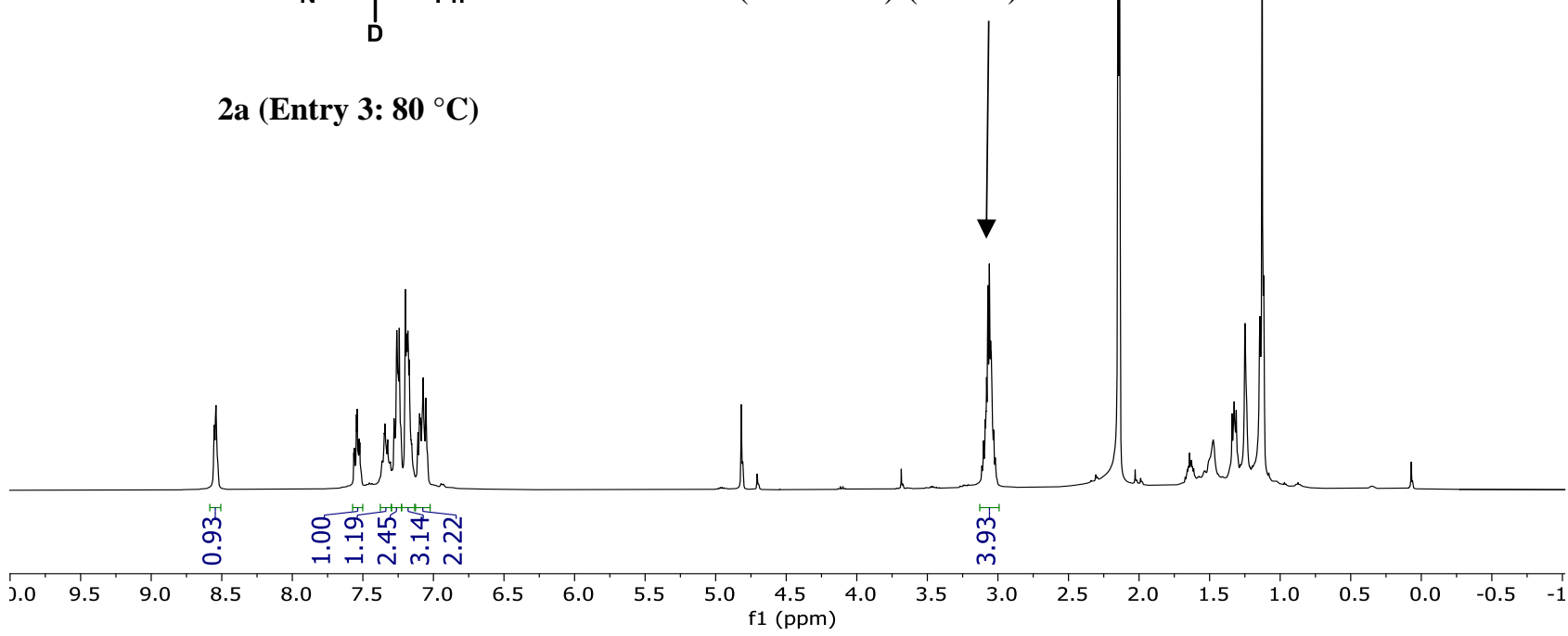

రั)

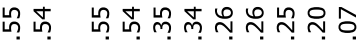

$\int_{\infty}^{\infty}$<smiles>[2H]C(Cc1ccccc1)c1ccccn1</smiles>

Deuteration:

$(4.28-3.94) /(4.28 / 2)=16 \%$

2a (Entry 4: $\left.120{ }^{\circ} \mathrm{C}\right)$

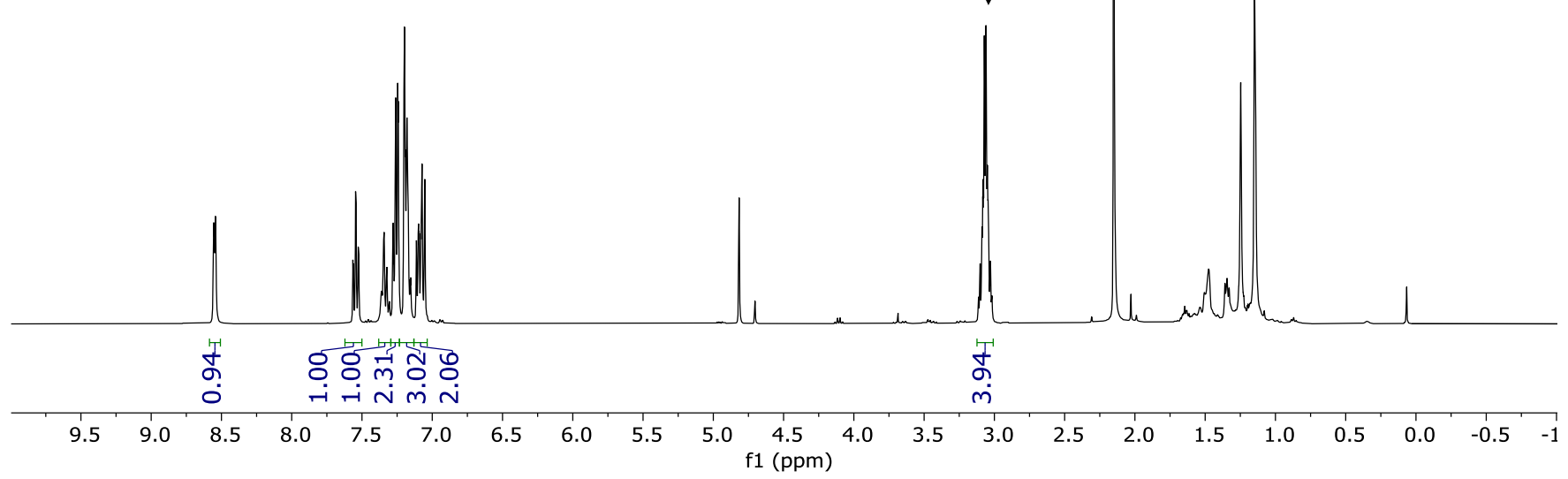

SI-11 
Procedure and Characterization Data for Time-Dependent Studies

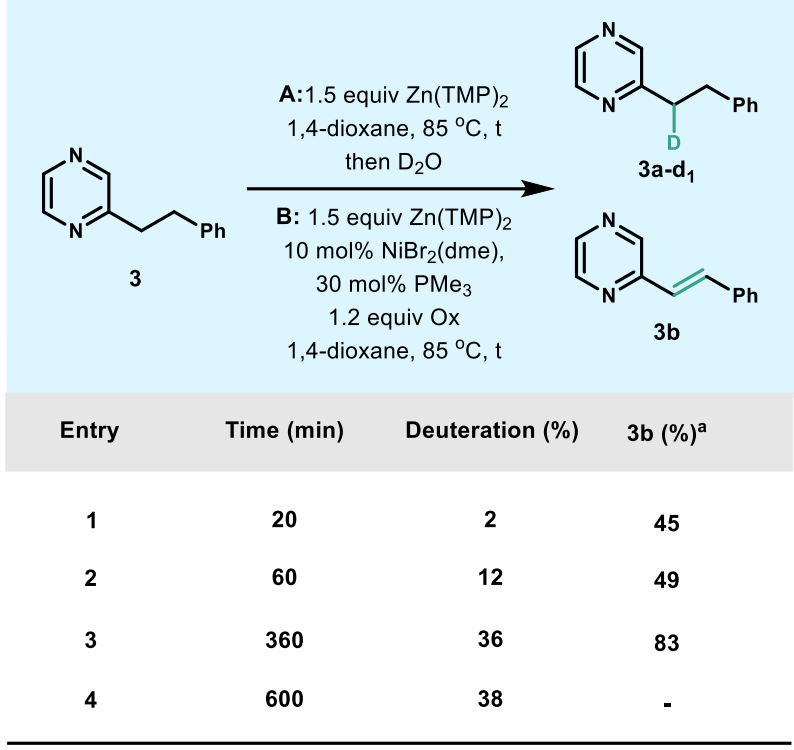

[a] Yield of dehydrogenation was determined by ${ }^{1} \mathrm{H}-\mathrm{NMR}$ analysis using 1,3,5trimethoxybenzene as an internal standard. Ox $=2$-bromo-5-methylthiophene 


\section{2-phenethylpyrazine (3)}<smiles>c1ccc(CCc2cnccn2)cc1</smiles>

Compound 3 was prepared according to known procedure, ${ }^{2}$ and the characterization data match those previously reported in the literature.

3

${ }^{1} \mathbf{H}$ NMR $\left(400 \mathrm{MHz}, \mathrm{CDCl}_{3}\right): \delta 8.52(\mathrm{~s}, 1 \mathrm{H}), 8.40(\mathrm{~d}, J=2.5 \mathrm{~Hz}, 1 \mathrm{H}), 8.36(\mathrm{~d}, J=1.5 \mathrm{~Hz}, 1 \mathrm{H}), 7.30-$ $7.24(\mathrm{~m}, 2 \mathrm{H}), 7.21-7.17(\mathrm{~m}, 3 \mathrm{H}), 3.15-3.05(\mathrm{~m}, 4 \mathrm{H})$

${ }^{13} \mathrm{C}$ NMR (101 MHz, $\left.\mathrm{CDCl}_{3}\right): \delta 156.9,144.8,144.2,142.5,140.9,128.6,128.5,126.4,37.3,35.5$

ESI-HRMS (m/z): $[\mathrm{M}+\mathrm{H}]^{+}$calc'd for $\mathrm{C}_{12} \mathrm{H}_{13} \mathrm{~N}^{+}$: 185.1073 ; found: 185.1079
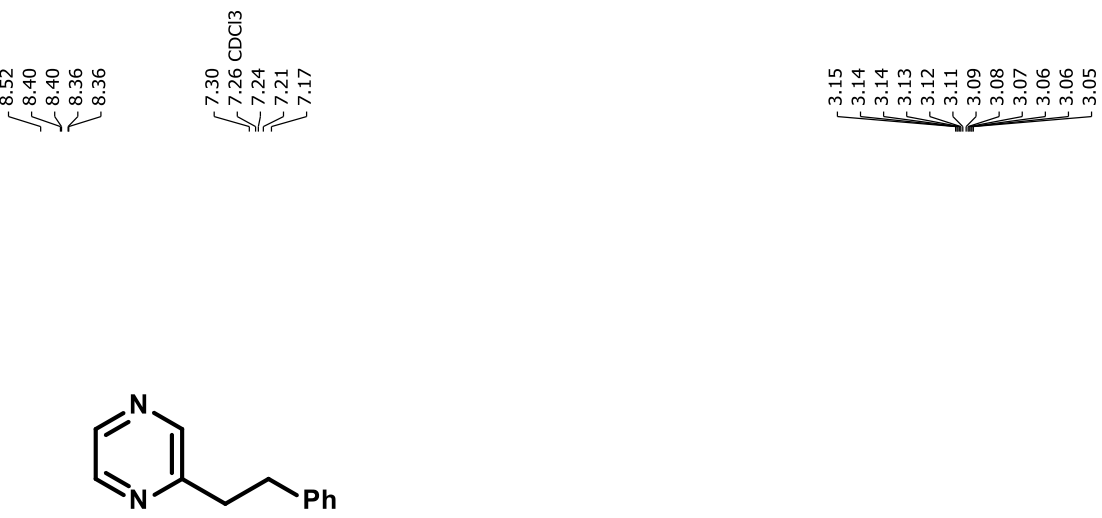

3

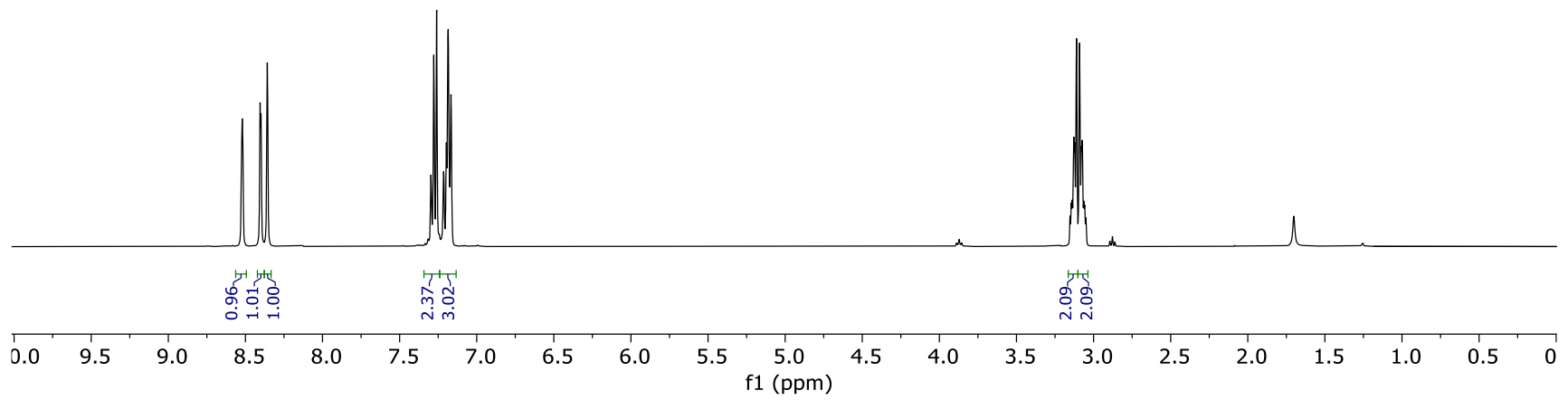




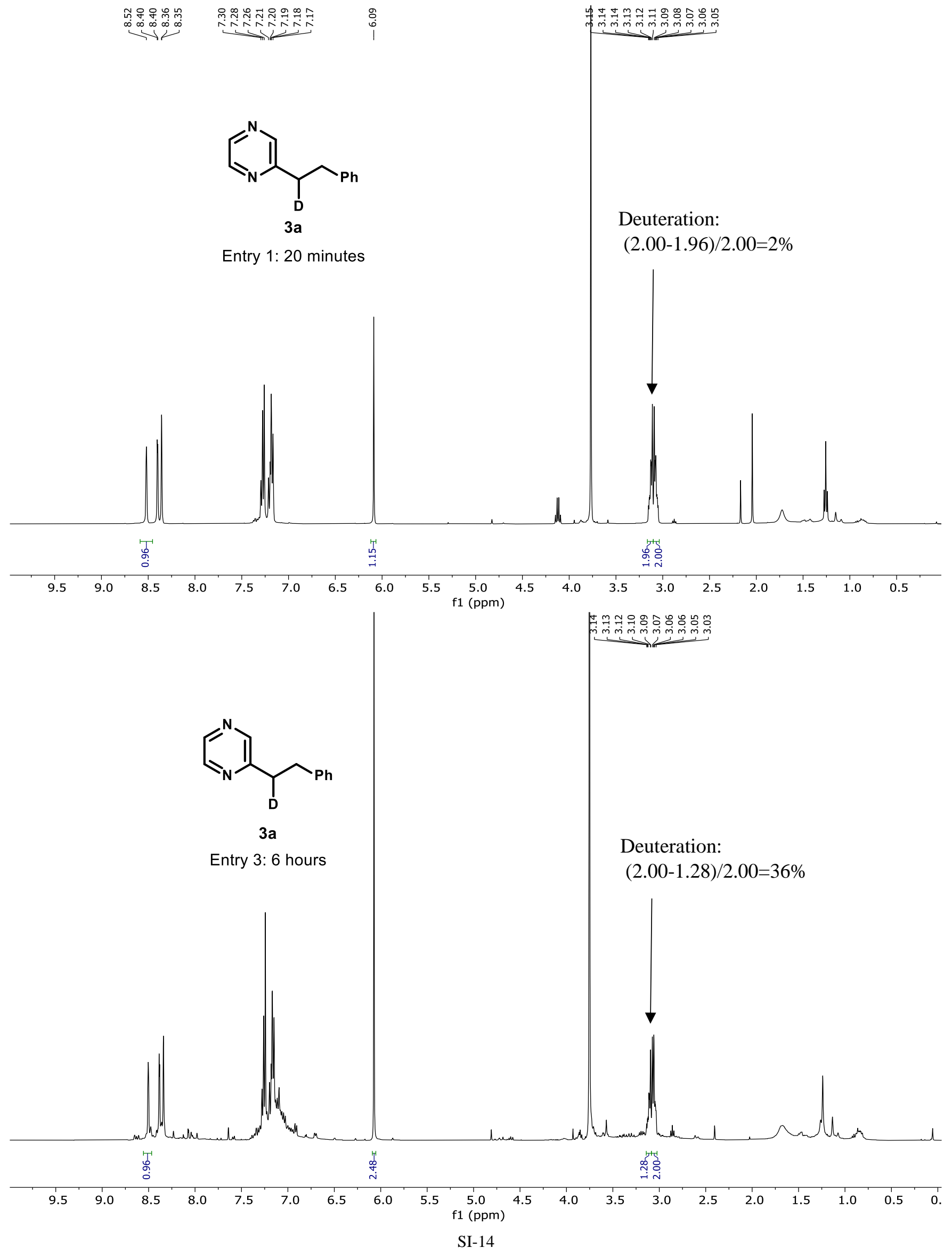




\section{Procedure and Characterization Data for Substrate Scope}

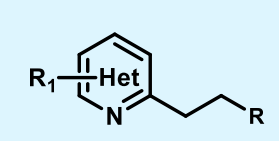

1-16

$\mathrm{F}_{3} \mathrm{C}$<smiles>Cc1ccc(CCc2ccccc2)nc1</smiles>

$6 \%(56 \%)$

1<smiles>N#Cc1ccc(CCc2ccccc2)nc1</smiles>

$-(36 \%)$
6<smiles>c1ccc2nc3c(cc2c1)CCCC3</smiles>

$28 \%(79 \%)$

10

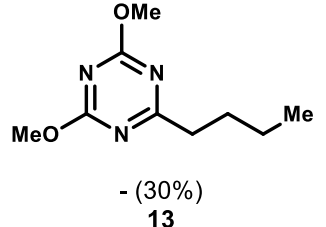

$\mathrm{A}: 1.5$ equiv $\mathrm{Zn}(\mathrm{TMP})_{2}$

$85^{\circ} \mathrm{C}, 4 \mathrm{~h}$, then $\mathrm{D}_{2} \mathrm{O}$

B: 1.5 equiv $\mathrm{Zn}(\mathrm{TMP})_{2}$ $\mathrm{NiBr}_{2}$ (dme)

$\mathrm{PMe}_{3}, \mathrm{TBAI}, \mathrm{Ox}$

dioxane, $85^{\circ} \mathrm{C}, 4 \mathrm{~h}$

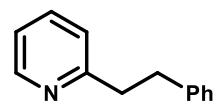

$22 \%(77 \%)$
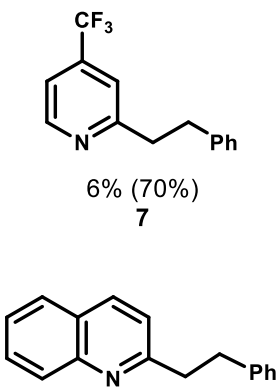

$12 \%(78 \%)$

11

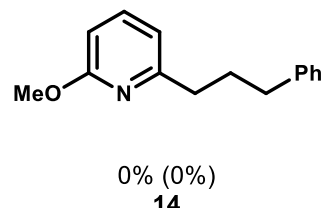

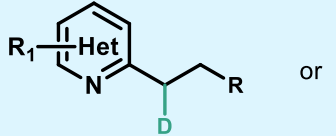

1a-16a

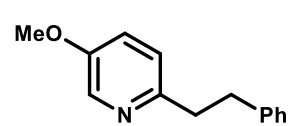

$0 \%(72 \%)$<smiles>c1ccc(CC2CCCCC2)nc1</smiles>

$0 \%(86 \%)$
8

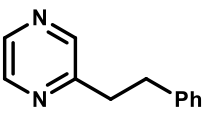

$36 \%(83 \%)$

3

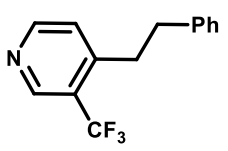

$0 \%(0 \%)$
15

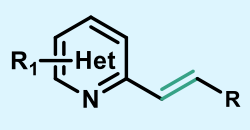

$1 b-16 b$

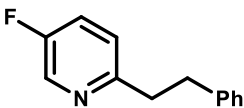

$5 \%(66 \%)$

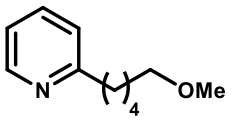

$0 \%(68 \%)$

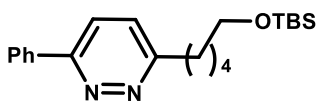

$29 \%(83 \%)$

12<smiles>c1cc2c(cn1)CCCC2</smiles>

$0 \%(0 \%)$

[a] Deuteration percentage was determined by crude ${ }^{1} \mathrm{H}$ NMR. [b] Isolated Yield of Ni-catalyzed dehydrogenation was shown in parentheses; Ox $=2$-bromo-5-methylthiophene for condition $\mathrm{B}$. [c] compounds $\mathbf{6}$ and 13 gave obvious degradation.

\section{General Procedure E for deprotonation of substrate scope (condition A):}

A flame-dried microwave vial equipped with a magnetic stir bar was evacuated and backfilled with $\mathrm{N}_{2}$ (this process was repeated three times). To the reaction vessel was added a solution of starting material $(0.1 \mathrm{mmol}, 1$ equiv.) in anhydrous 1,4-dioxane $(0.1 \mathrm{M}, 1 \mathrm{~mL})$, followed by the commercial solution of $\mathrm{Zn}(\mathrm{TMP})_{2}\left(0.3 \mathrm{~mL}, 0.5 \mathrm{M}\right.$ in toulene, $0.15 \mathrm{mmol}, 1.5$ equiv). The reaction was stirred at $85{ }^{\circ} \mathrm{C}$ for 4 hours.

Immediately after removal from heat, the reaction was quenched with $\mathrm{D}_{2} \mathrm{O}(0.5 \mathrm{~mL})$ and stirred for 5 minutes to cool down to room temperature. A solution of internal standard 1,3,5-trimethoxybenzene in EtOAc was added ( $0.33 \mathrm{mmol}$ unless otherwise stated) before extraction with EtOAc $(4 \times 1.5 \mathrm{~mL})$, and the combined organic extracts were dried with $\mathrm{Na}_{2} \mathrm{SO}_{4}$ and filtered through a short silica plug and washed with $\mathrm{EtOAc}(5 \mathrm{~mL})$ and concentrated under reduced pressure by rotary evaporation. Then $0.2 \mathrm{~mL}$ of $\mathrm{CDCl}_{3}$ 
was added and the excess solvent was removed under vacuum. Finally, the deuteration and degradation percentage was determined by crude ${ }^{1} \mathrm{H}$ NMR based on the internal standard.

\section{General Procedure F for dehydrogenation (condition B)²:}

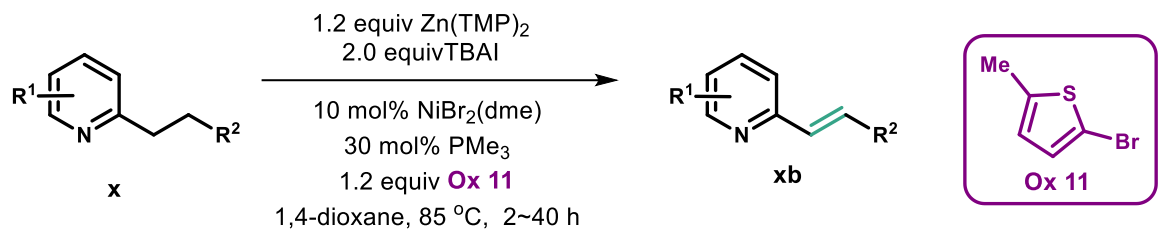

To a flame-dried microwave vial equipped with a magnetic stir bar was added starting material $\mathbf{1}$ ( $0.20 \mathrm{mmol}, 1.0$ equiv) and TBAI (148 mg, $0.40 \mathrm{mmol}, 2.0$ equiv). The reaction vessel was sealed, evacuated and backfilled with $\mathrm{N}_{2}$ (this process was repeated three times). To the reaction vessel was added anhydrous 1,4-dioxane $(1.5 \mathrm{~mL})$ and commercial $\mathrm{Zn}(\mathrm{TMP})_{2}(0.48 \mathrm{~mL}, 0.5 \mathrm{M}$ in toluene, $0.24 \mathrm{mmol}, 1.2$ equiv) at room temperature. Then to this mixture was added $0.5 \mathrm{~mL}$ of a stock solution containing $\mathrm{NiBr}_{2}(\mathrm{dme})(6.2 \mathrm{mg}, 0.02 \mathrm{mmol}, 10 \mathrm{~mol} \%), \mathrm{PMe}_{3}(0.06 \mathrm{~mL}, 0.06 \mathrm{mmol}, 30 \mathrm{~mol} \%$ ), and 2-bromo-5methylthiophene $(27.4 \mathrm{uL}, 0.24 \mathrm{mmol}, 1.2$ equiv) in 1,4-dioxane. The reaction vessel was placed into a pre-heated $85^{\circ} \mathrm{C}$ oil bath, and stirred until complete consumption of starting material as determined by TLC analysis. (Note: more reproducible results can be obtained using the sequential addition procedure.)

The reaction mixture was cooled to ambient temperature and quenched by the addition of sat. aq. $\mathrm{NH}_{4} \mathrm{Cl}(5 \mathrm{~mL})$. The reaction mixture was diluted with EtOAc $(5 \mathrm{~mL})$ and the organic phase was separated. The aqueous phase was extracted with EtOAc $(3 \times 5 \mathrm{~mL})$ and the combined organic extracts were washed with brine $(5 \mathrm{~mL})$, dried over anhydrous $\mathrm{Na}_{2} \mathrm{SO}_{4}$, filtered, and concentrated under reduced pressure by rotary evaporation. The crude mixture was purified by flash column chromatography on silica gel.

\section{5-methoxy-2-phenethylpyridine (4)}

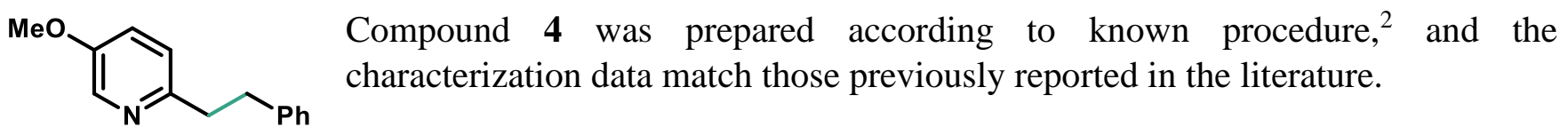

${ }^{1} \mathbf{H}$ NMR $\left(400 \mathrm{MHz}, \mathrm{CDCl}_{3}\right): \delta 8.27(\mathrm{~d}, J=3.0 \mathrm{~Hz}, 1 \mathrm{H}), 7.29-7.25(\mathrm{~m}, 2 \mathrm{H}), 7.22-7.16(\mathrm{~m}, 3 \mathrm{H}), 7.09$ $(\mathrm{dd}, J=8.5,3.0 \mathrm{~Hz}, 1 \mathrm{H}), 6.99(\mathrm{~d}, J=8.5 \mathrm{~Hz}, 1 \mathrm{H}), 3.83(\mathrm{~s}, 3 \mathrm{H}), 3.06-3.01(\mathrm{~m}, 4 \mathrm{H})$

${ }^{13} \mathrm{C}$ NMR (101 $\left.\mathrm{MHz}_{2} \mathrm{CDCl}_{3}\right): \delta 154.0,153.4,141.8,136.7,128.6,128.4,126.0,123.1,121.2,55.7,39.3$, 36.4

ESI-HRMS (m/z): $[\mathrm{M}+\mathrm{H}]^{+}$calc'd for $\mathrm{C}_{14} \mathrm{H}_{16} \mathrm{NO}^{+}$: 214.1226 ; found: 214.1232 


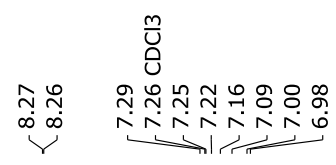

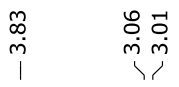

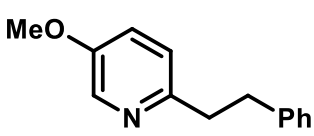

4

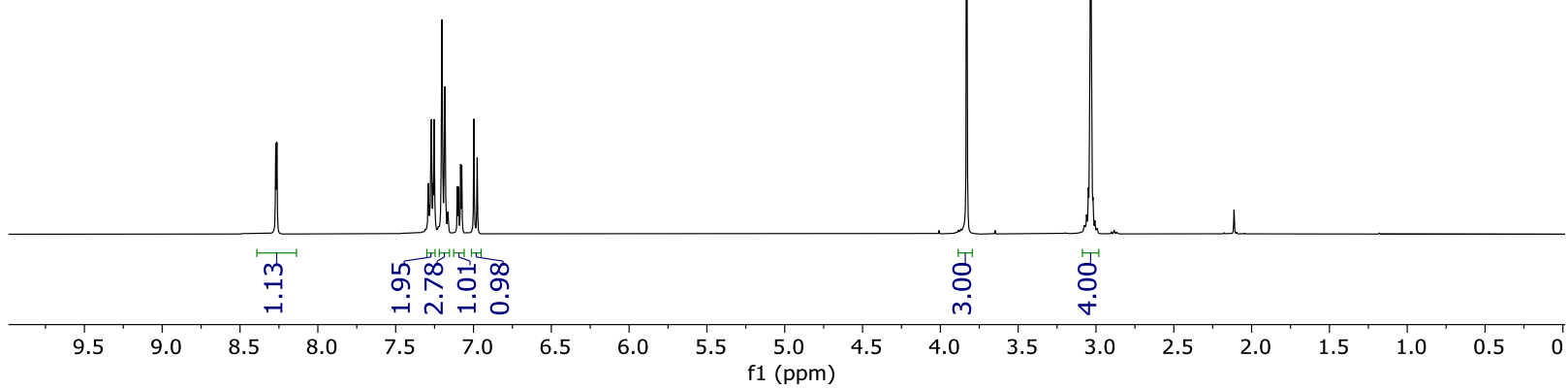

บิ

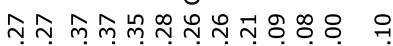

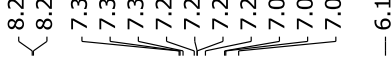

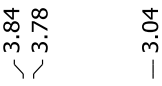

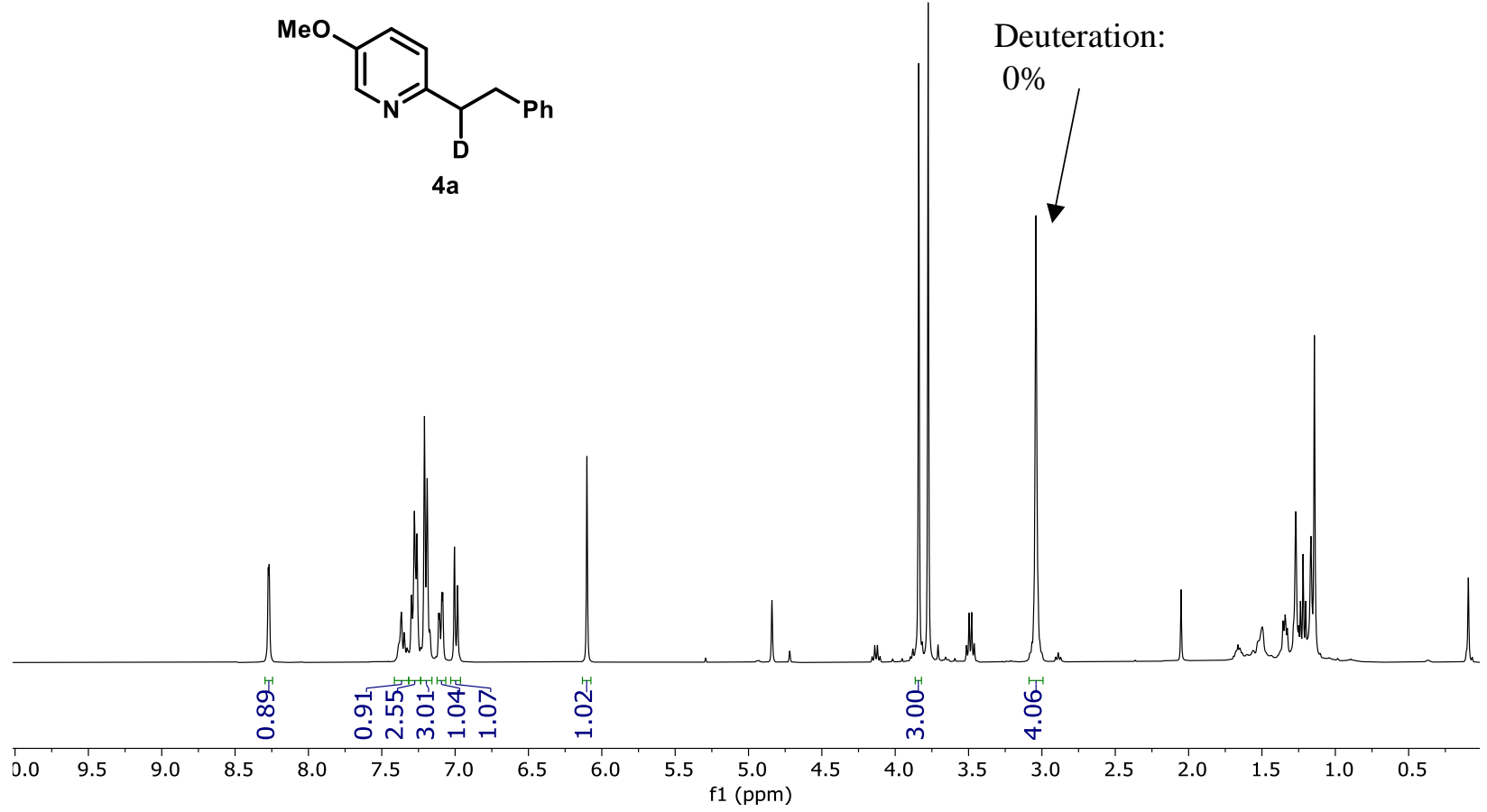

SI-17 


\section{5-fluoro-2-phenethylpyridine (5)}

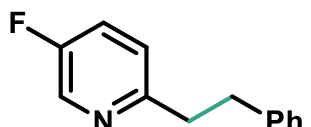

Compound 5 was prepared according to known procedure, ${ }^{2}$ and the characterization data match those previously reported in the literature.

${ }^{1} \mathbf{H}$ NMR $\left(400 \mathrm{MHz}, \mathrm{CDCl}_{3}\right): \delta 8.43(\mathrm{~d}, J=3.0 \mathrm{~Hz}, 1 \mathrm{H}), 7.31-7.25(\mathrm{~m}, 3 \mathrm{H}), 7.22-7.15(\mathrm{~m}, 3 \mathrm{H}), 7.05$ $(\mathrm{dd}, J=8.6,4.4 \mathrm{~Hz}, 1 \mathrm{H}), 3.13-3.03(\mathrm{~m}, 4 \mathrm{H})$

${ }^{13}$ C NMR (101 MHz, $\left.\mathrm{CDCl}_{3}\right): \delta 158.2\left(\mathrm{~d}, J_{C-F}=253.4 \mathrm{~Hz}\right), 157.2\left(\mathrm{~d}, J_{C-F}=3.9 \mathrm{~Hz}\right), 141.4,137.4\left(\mathrm{~d}, J_{C-F}\right.$ $=23.1 \mathrm{~Hz}), 128.6,128.5,126.1,123.7\left(\mathrm{~d}, J_{C-F}=3.9 \mathrm{~Hz}\right), 123.1\left(\mathrm{~d}, J_{C-F}=18.1 \mathrm{~Hz}\right), 39.4,36.1$

${ }^{19}$ F NMR $\left(376 \mathrm{MHz}, \mathrm{CDCl}_{3}\right): \delta-131.2(\mathrm{q}, J=3.8 \mathrm{~Hz})$

ESI-HRMS (m/z): $[\mathrm{M}+\mathrm{H}]^{+}$calc'd for $\mathrm{C}_{13} \mathrm{H}_{13} \mathrm{FN}^{+}$: 202.1027; found: 202.1032 

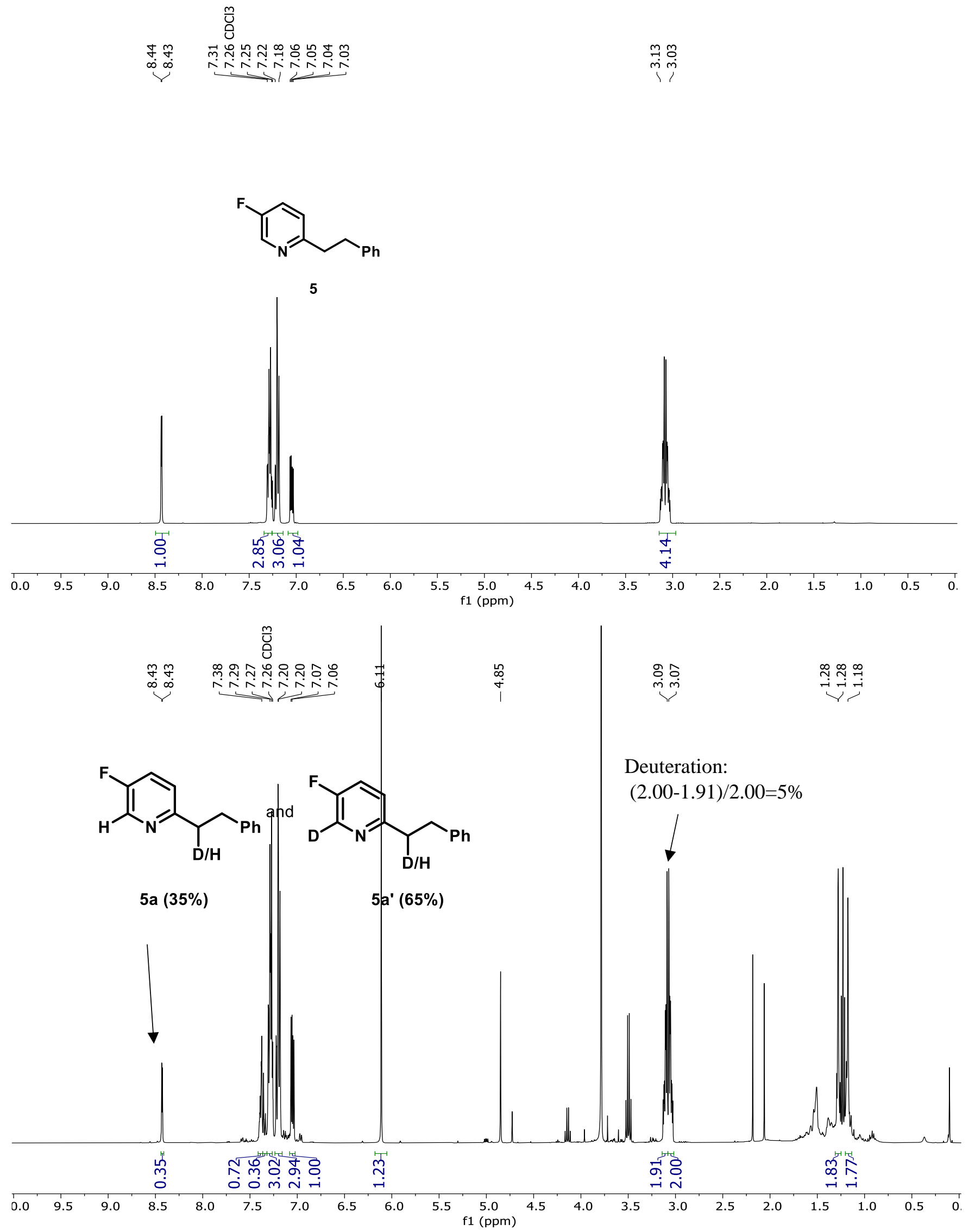


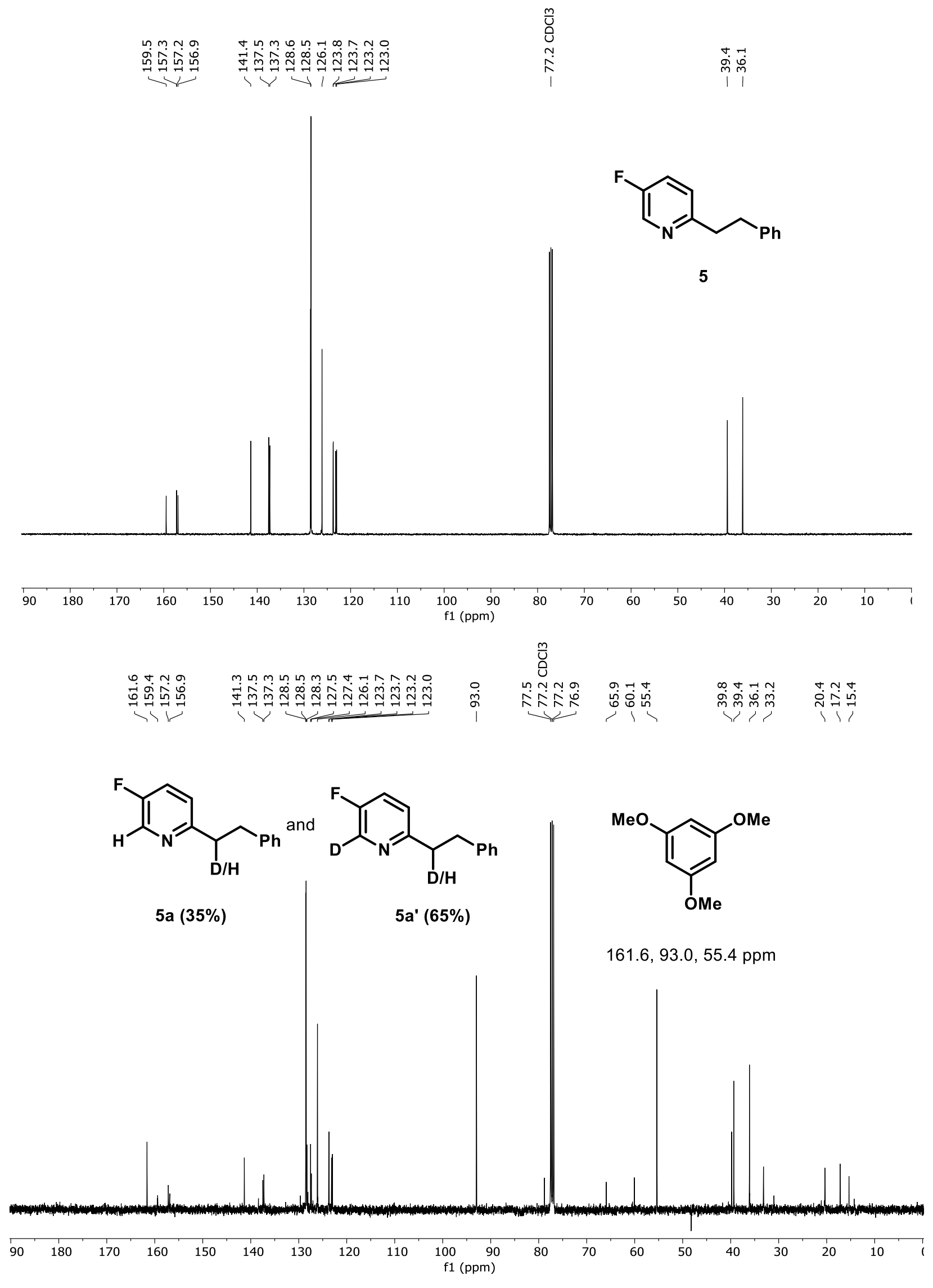




\section{6-phenethylnicotinonitrile (6)}

NC Compound 6 was prepared according to known procedure, ${ }^{2}$ and the characterization data match those previously reported in the literature.

${ }^{1} \mathbf{H}$ NMR $\left(400 \mathrm{MHz}, \mathrm{CDCl}_{3}\right): \delta 8.84(\mathrm{~d}, J=1.7 \mathrm{~Hz}, 1 \mathrm{H}), 7.81(\mathrm{dd}, J=8.1,2.2 \mathrm{~Hz}, 1 \mathrm{H}), 7.29(\mathrm{dd}, J=8.0$, $6.5 \mathrm{~Hz}, 2 \mathrm{H}), 7.28-7.17(\mathrm{~m}, 4 \mathrm{H}), 3.20-3.16(\mathrm{~m}, 2 \mathrm{H}), 3.10-3.05(\mathrm{~m}, 2 \mathrm{H})$

${ }^{13} \mathrm{C}$ NMR (101 MHz, $\left.\mathrm{CDCl}_{3}\right): \delta 165.9,152.3,140.6,139.3,128.6,128.5,126.4,123.2,117.0,107.5,40.4$, 35.4

ESI-HRMS (m/z): [M+H] $]^{+}$calc'd for $\mathrm{C}_{14} \mathrm{H}_{13} \mathrm{~N}_{2}{ }^{+}$: 209.1073; found: 209.1079 


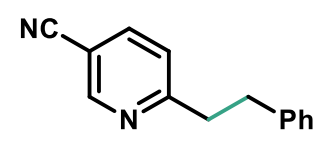

6
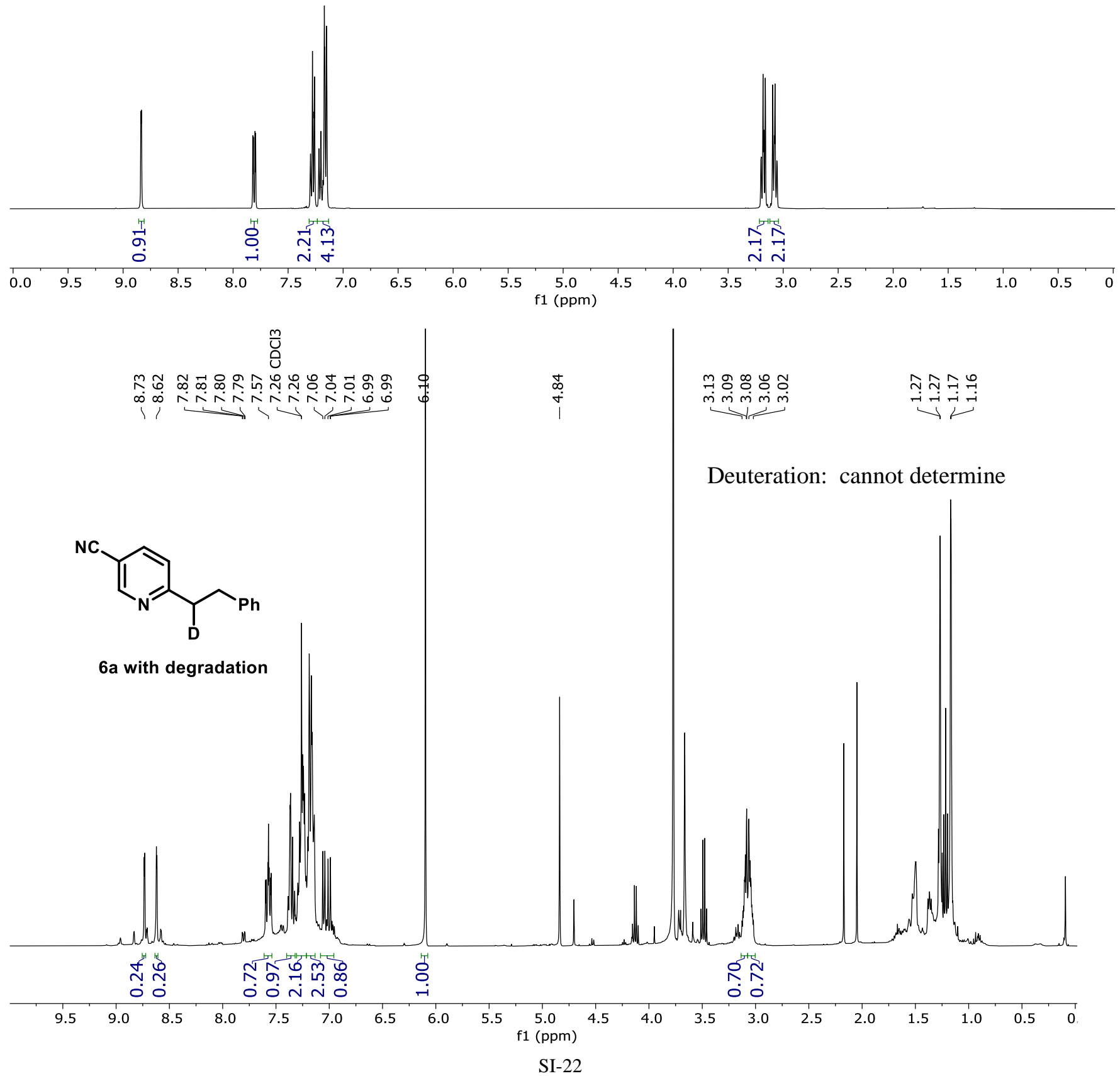


\section{2-phenethyl-4-(trifluoromethyl)pyridine (7)}

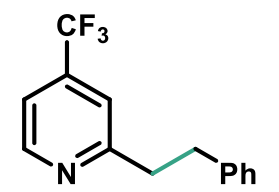

Compound 7 was prepared according to known procedure, ${ }^{2}$ and the characterization data match those previously reported in the literature.

${ }^{1} \mathbf{H}$ NMR $\left(400 \mathrm{MHz}, \mathrm{CDCl}_{3}\right): \delta 8.85(\mathrm{~s}, 1 \mathrm{H}), 7.79(\mathrm{dd}, J=8.2,2.4 \mathrm{~Hz}, 1 \mathrm{H}), 7.32-7.27(\mathrm{~m}, 2 \mathrm{H}), 7.24-$ $7.16(\mathrm{~m}, 4 \mathrm{H}), 3.21-3.16(\mathrm{~m}, 2 \mathrm{H}), 3.13-3.07(\mathrm{~m}, 2 \mathrm{H})$

${ }^{13} \mathrm{C}$ NMR (101 MHz, $\left.\mathrm{CDCl}_{3}\right): \delta 165.4,146.4\left(\mathrm{q}, J_{C-F}=4.1 \mathrm{~Hz}\right), 141.0,133.40\left(\mathrm{q}, J_{C-F}=3.5 \mathrm{~Hz}\right), 128.6$, $128.5,126.6,124.4\left(\mathrm{q}, J_{C-F}=33.0 \mathrm{~Hz}\right), 123.9\left(\mathrm{q}, J_{C-F}=272.1 \mathrm{~Hz}\right), 122.8,40.2,35.7$

${ }^{19}$ F NMR (376 MHz, $\left.\mathrm{CDCl}_{3}\right): \delta-62.2(\mathrm{~s})$

ESI-HRMS (m/z): $[\mathrm{M}+\mathrm{H}]^{+}$calc'd for $\mathrm{C}_{14} \mathrm{H}_{13} \mathrm{~F}_{3} \mathrm{~N}^{+}: 252.0995$; found: 252.1000 

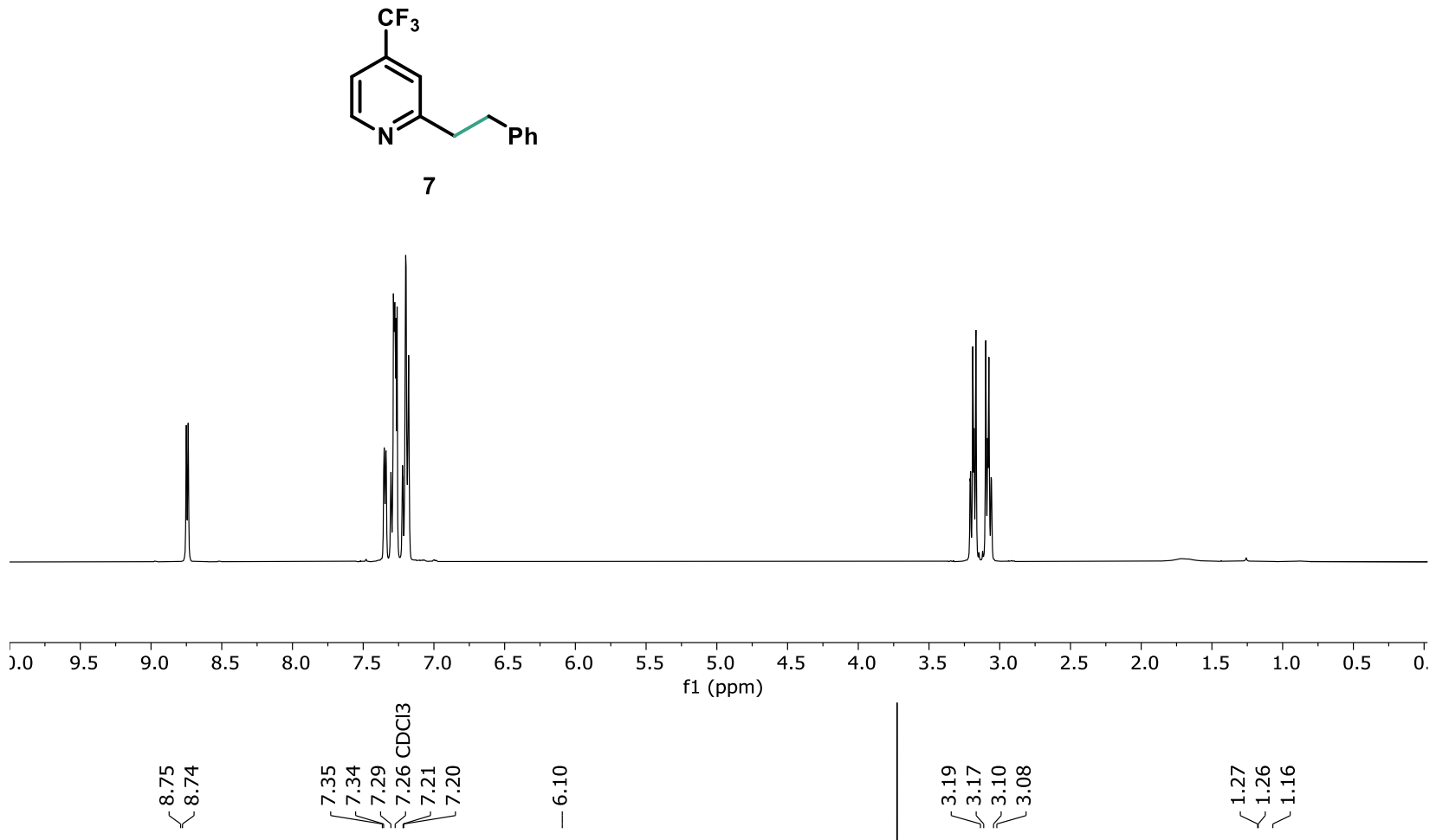<smiles>FC(F)(F)c1ccnc(CCc2ccccc2)c1</smiles>

$7 a$

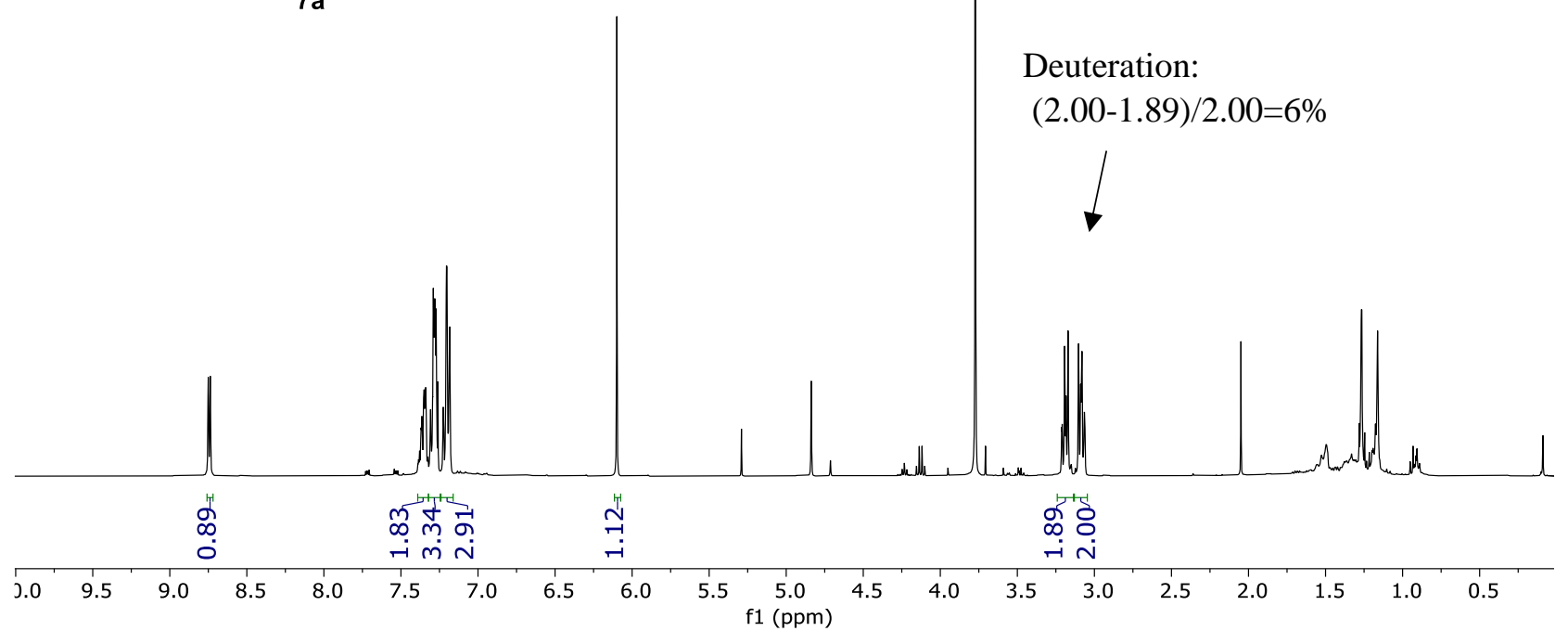




\section{2-(cyclohexylmethyl)pyridine (8)}<smiles>c1ccc(CC2CCCCC2)nc1</smiles>

Compound 8 was prepared according to known procedure, ${ }^{2}$ and the characterization data match those previously reported in the literature.

${ }^{1} \mathbf{H}$ NMR $\left(400 \mathrm{MHz}_{\mathrm{CDCl}}\right): \delta 8.52(\mathrm{~d}, J=4.8 \mathrm{~Hz}, 1 \mathrm{H}), 7.57-7.53(\mathrm{~m}, 1 \mathrm{H}), 7.09-7.05(\mathrm{~m}, 2 \mathrm{H}), 2.64$ $(\mathrm{d}, J=7.1 \mathrm{~Hz}, 2 \mathrm{H}), 1.81-1.72(\mathrm{~m}, 1 \mathrm{H}), 1.71-1.58(\mathrm{~m}, 5 \mathrm{H}), 1.27-1.09(\mathrm{~m}, 3 \mathrm{H}), 1.06-0.93(\mathrm{~m}, 2 \mathrm{H})$

${ }^{13}$ C NMR (101 MHz, $\left.\mathrm{CDCl}_{3}\right): \delta 161.5,149.4,136.0,123.7,120.9,46.5,38.8,33.3,26.6,26.4$

ESI-HRMS (m/z): $[\mathrm{M}+\mathrm{H}]^{+}$calc'd for $\mathrm{C}_{12} \mathrm{H}_{18} \mathrm{~N}^{+}$: 176.1434; found: 176.1439 


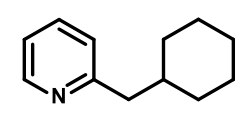

8
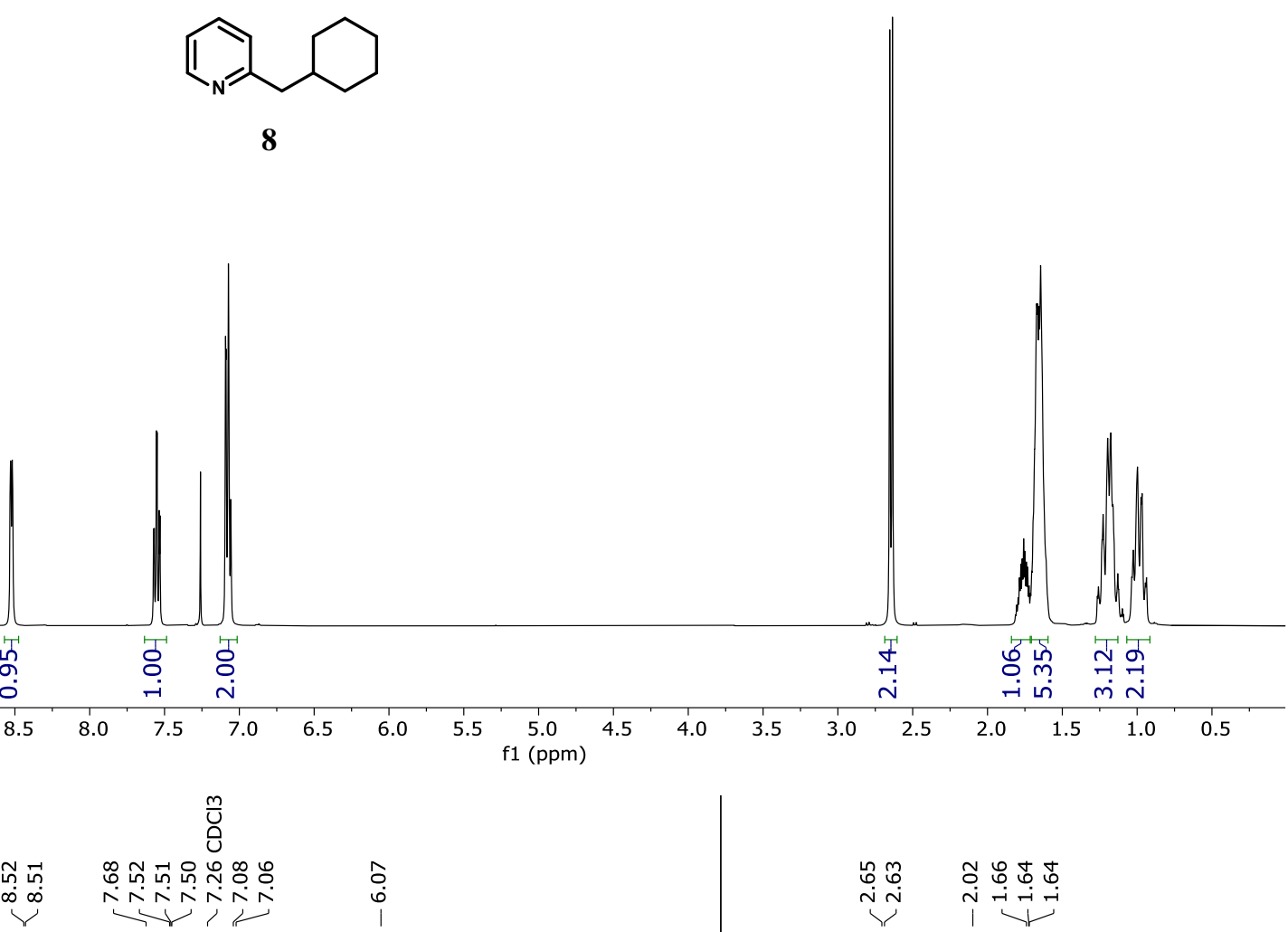<smiles>[2H]C(c1ccccn1)C1CCCCC1</smiles>

نِ

$8 \mathbf{a}$

Deuteration $<1 \%$

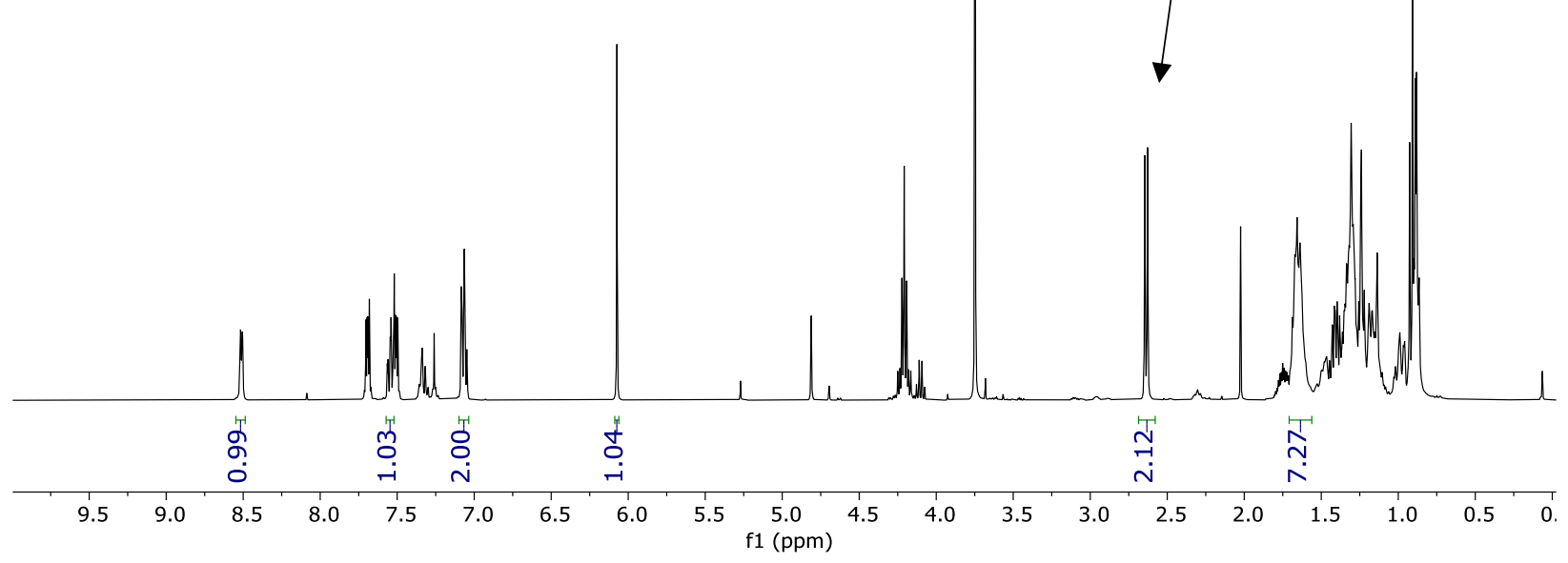




\section{2-(5-methoxypentyl)pyridine (9)}

Compound 9 was prepared according to known procedure, ${ }^{2}$ and the

${ }^{1} \mathbf{H}$ NMR $\left(400 \mathrm{MHz}, \mathrm{CDCl}_{3}\right): \delta 8.50(\mathrm{~d}, J=4.0 \mathrm{~Hz}, 1 \mathrm{H}), 7.58-7.53(\mathrm{~m}, 1 \mathrm{H}), 7.14-7.05(\mathrm{~m}, 2 \mathrm{H}), 3.35$ $(\mathrm{t}, J=6.6 \mathrm{~Hz}, 2 \mathrm{H}), 3.30(\mathrm{~s}, 3 \mathrm{H}), 2.88-2.73(\mathrm{~m}, 2 \mathrm{H}), 1.78-1.70(\mathrm{~m}, 2 \mathrm{H}), 1.63-1.56(\mathrm{~m}, 2 \mathrm{H}), 1.44-$ $1.36(\mathrm{~m}, 2 \mathrm{H})$

${ }^{13} \mathrm{C}$ NMR (101 MHz, $\left.\mathrm{CDCl}_{3}\right): \delta 162.4,149.3,136.3,122.8,121.0,72.9,58.6,38.5,29.8,29.6,26.1$

ESI-HRMS (m/z): $[\mathrm{M}+\mathrm{H}]^{+}$calc'd for $\mathrm{C}_{11} \mathrm{H}_{18} \mathrm{NO}^{+}$: 180.1383 ; found: 180.1388 


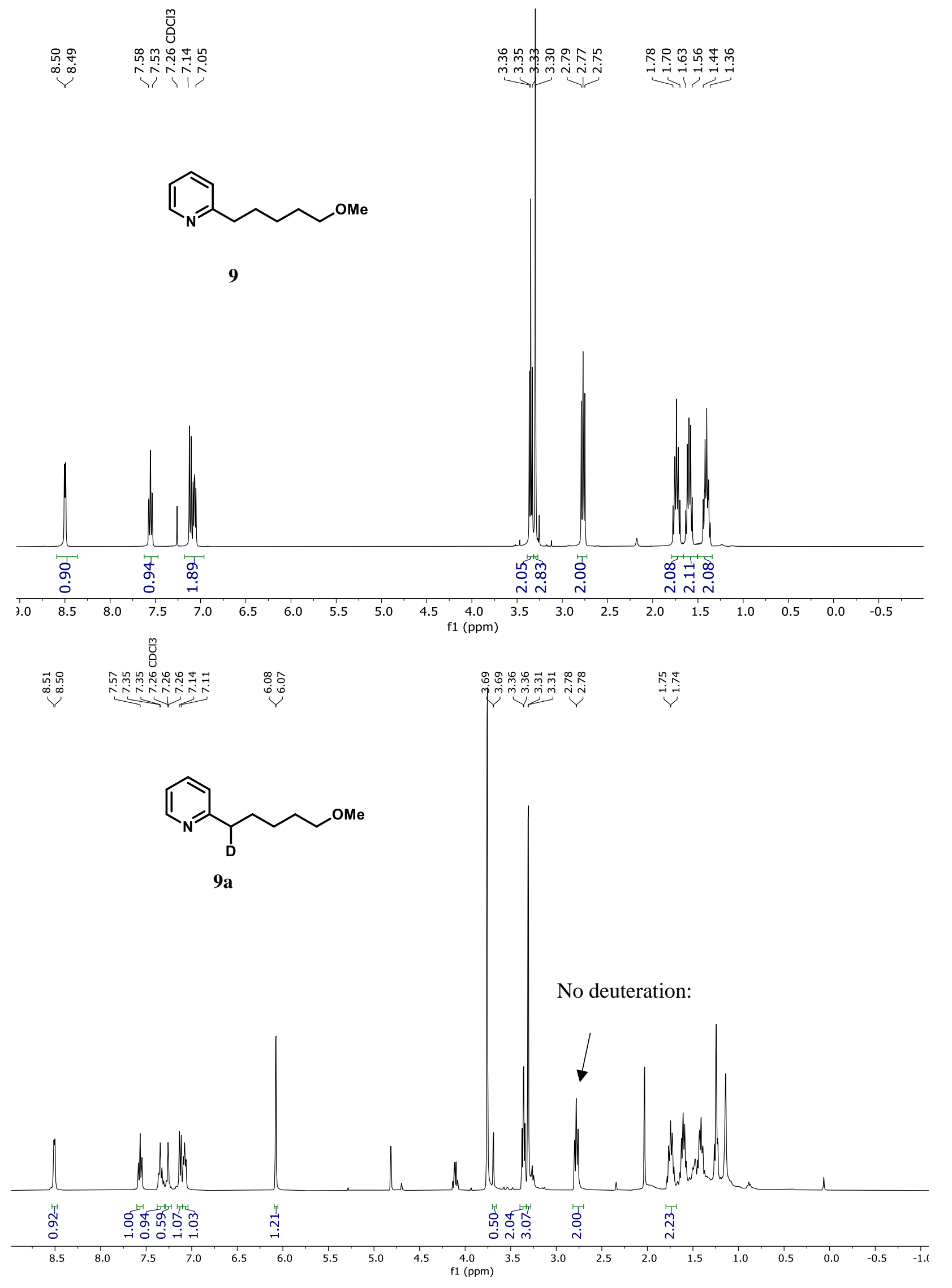




\section{1,2,3,4-tetrahydroacridine (10)}

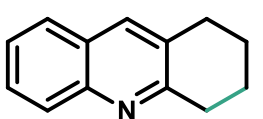

Compound 10 was prepared according to known procedure, ${ }^{3}$ and the characterization data match those previously reported in the literature.

${ }^{1} \mathbf{H}$ NMR $\left(400 \mathrm{MHz}, \mathrm{CDCl}_{3}\right) \delta 7.97(\mathrm{~d}, J=8.5 \mathrm{~Hz}, 1 \mathrm{H}), 7.76(\mathrm{~s}, 1 \mathrm{H}), 7.67(\mathrm{dd}, J=8.2,1.5 \mathrm{~Hz}, 1 \mathrm{H}), 7.59$ $(\mathrm{t}, J=7.7 \mathrm{~Hz}, 1 \mathrm{H}), 7.46-7.36(\mathrm{~m}, 1 \mathrm{H}), 3.12(\mathrm{t}, J=6.5 \mathrm{~Hz}, 2 \mathrm{H}), 2.95(\mathrm{t}, J=6.4 \mathrm{~Hz}, 2 \mathrm{H}), 2.07-1.92$ (m, 2H), $1.92-1.82(\mathrm{~m}, 2 \mathrm{H})$.

${ }^{13} \mathrm{C}$ NMR $\left(101 \mathrm{MHz}, \mathrm{CDCl}_{3}\right) \delta 159.4,146.8,135.1,131.1,128.6,128.4,127.3,127.0,125.6,33.7,29.4$, 23.4, 23.0.

GC-MS (m/z): $[\mathrm{M}+\mathrm{H}]^{+}$calc'd for $\mathrm{C}_{13} \mathrm{H}_{13} \mathrm{~N}^{+}$: 183.1; found: 183.1 


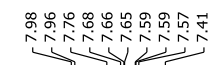

ทู่نู<smiles>c1ccc2nc3c(cc2c1)CCCC3</smiles>

10
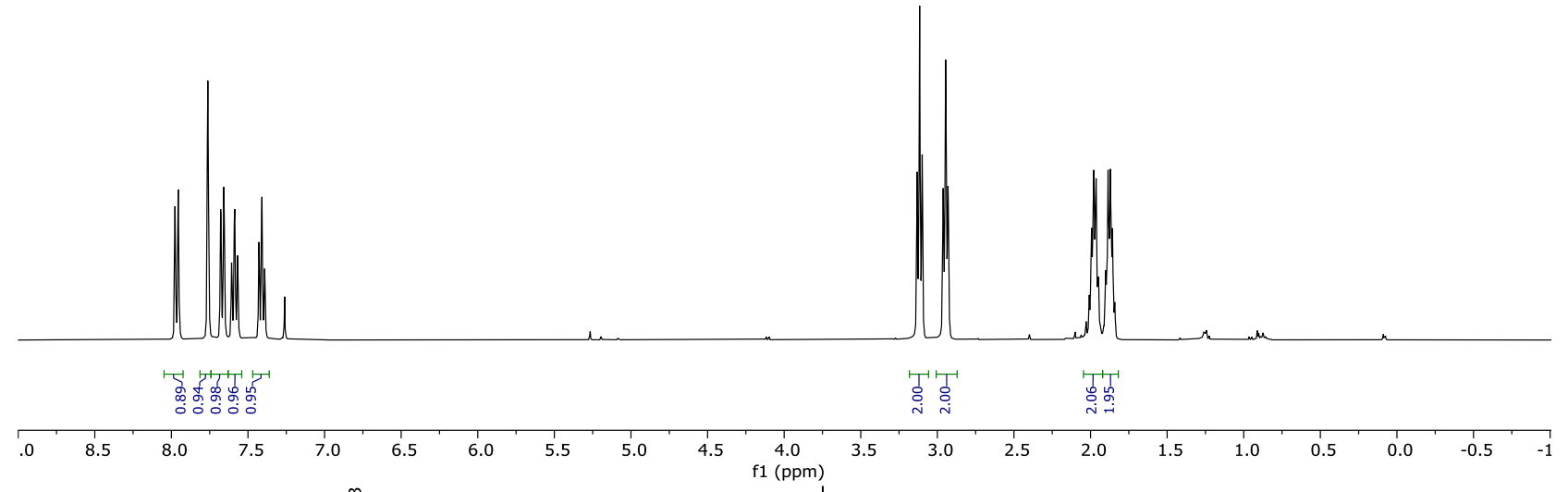
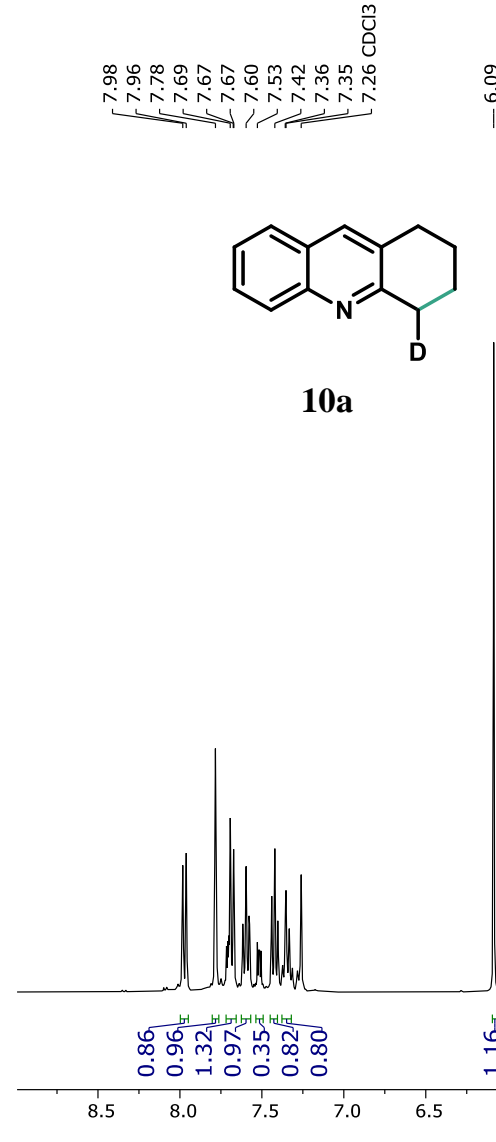

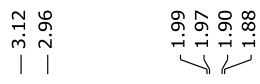

Deuteration:

$(2.00-1.44) / 2.00=28 \%$
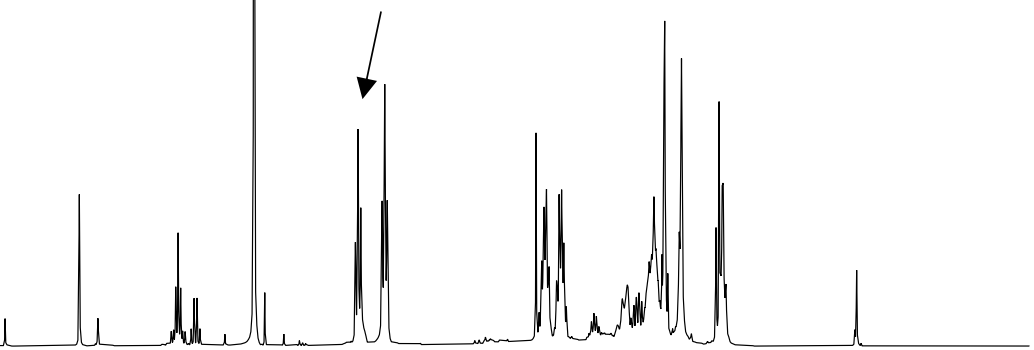

尔管

लूँ बूँ

2.0

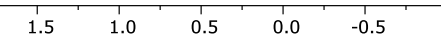




\section{2-phenethylquinoline (11)}

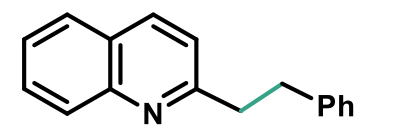

Compound 11 was prepared according to known procedure, ${ }^{2}$ and the characterization data match those previously reported in the literature.

${ }^{1} \mathbf{H}$ NMR $\left(400 \mathrm{MHz}, \mathrm{CDCl}_{3}\right): \delta 8.10-8.04(\mathrm{~m}, 2 \mathrm{H}), 7.79(\mathrm{~d}, J=8.1 \mathrm{~Hz}, 1 \mathrm{H}), 7.73-7.69(\mathrm{~m}, 1 \mathrm{H}), 7.52-$ $7.48(\mathrm{~m}, 1 \mathrm{H}), 7.31-7.18(\mathrm{~m}, 6 \mathrm{H}), 3.33-3.28(\mathrm{~m}, 2 \mathrm{H}), 3.20-3.15(\mathrm{~m}, 2 \mathrm{H})$

${ }^{13} \mathbf{C}$ NMR $\left(101 \mathrm{MHz}, \mathrm{CDCl}_{3}\right): \delta 161.9,148.1,141.7,136.4,129.5,129.0,128.7,128.5,127.7,126.9$, $126.1,125.9,121.7,41.1,36.1$

ESI-HRMS (m/z): $[\mathrm{M}+\mathrm{H}]^{+}$calc'd for $\mathrm{C}_{17} \mathrm{H}_{16} \mathrm{~N}^{+}: 234.1277$; found: 234.1283 


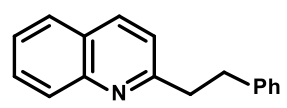

11
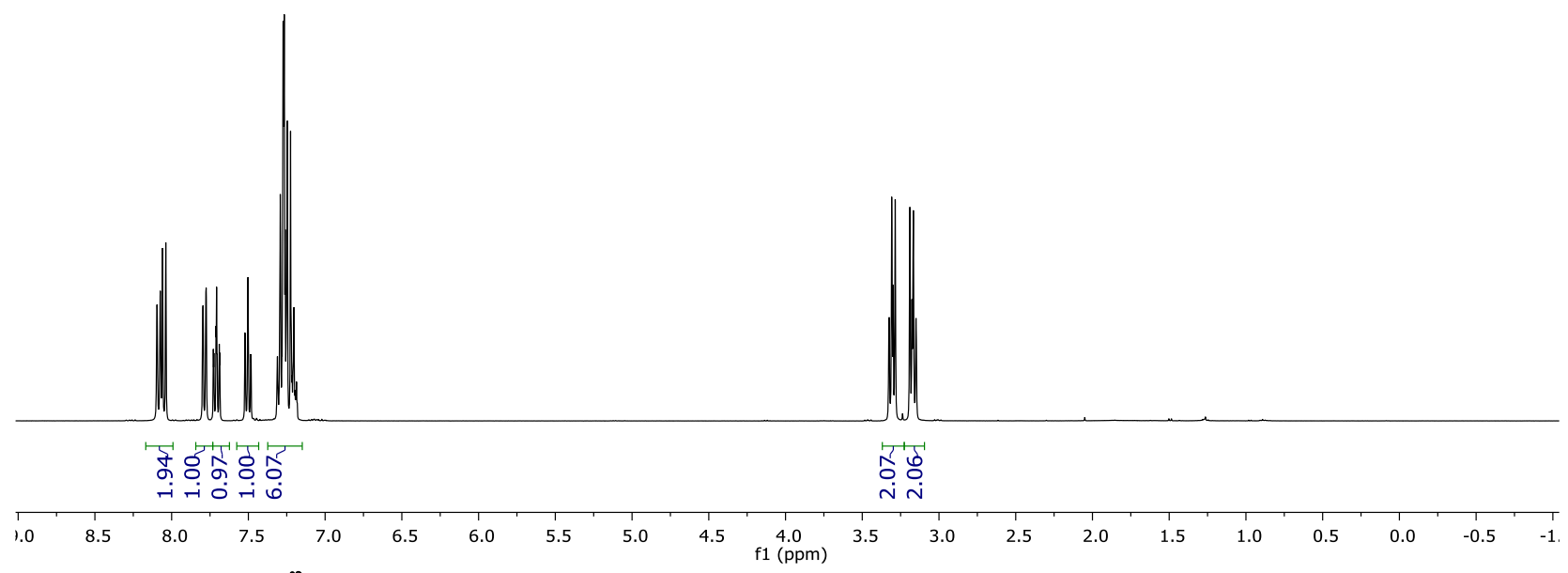
บิ

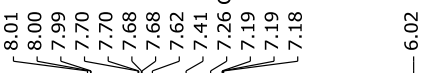

ํํำำㅇํำ
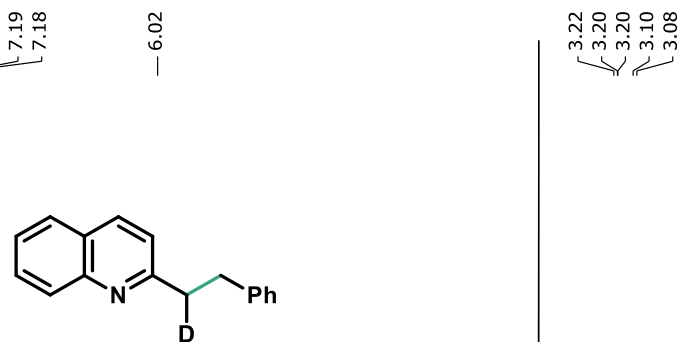

11a

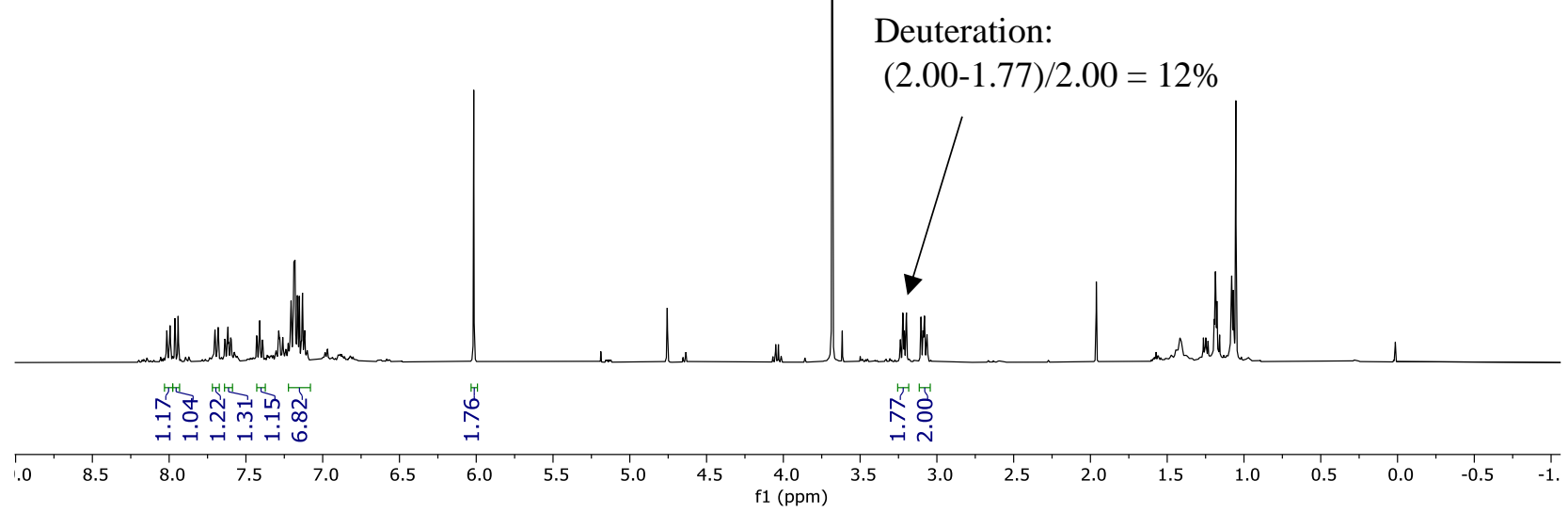




\section{3-(5-((tert-butyldimethylsilyl)oxy)pentyl)-6-phenylpyridazine (12)}

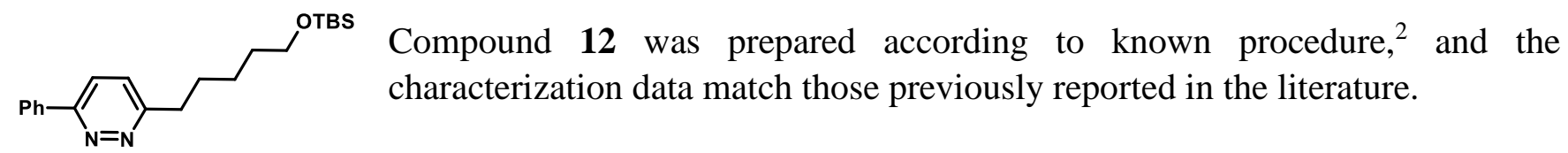

${ }^{1} \mathbf{H}$ NMR $\left(400 \mathrm{MHz}, \mathrm{CDCl}_{3}\right): \delta 8.08(\mathrm{~d}, J=6.7 \mathrm{~Hz}, 2 \mathrm{H}), 7.77(\mathrm{~d}, J=8.7 \mathrm{~Hz}, 1 \mathrm{H}), 7.54-7.45(\mathrm{~m}, 3 \mathrm{H})$, $7.37(\mathrm{~d}, J=8.7 \mathrm{~Hz}, 1 \mathrm{H}), 3.60(\mathrm{t}, J=6.4 \mathrm{~Hz}, 2 \mathrm{H}), 3.02(\mathrm{t}, J=6.4 \mathrm{~Hz}, 2 \mathrm{H}), 1.90-1.80(\mathrm{~m}, 2 \mathrm{H}), 1.56-$ $1.47(\mathrm{~m}, 2 \mathrm{H}), 1.47-1.35(\mathrm{~m}, 4 \mathrm{H}), 0.89(\mathrm{~s}, 9 \mathrm{H}), 0.04(\mathrm{~s}, 6 \mathrm{H})$

${ }^{13} \mathrm{C}$ NMR (101 MHz, $\left.\mathrm{CDCl}_{3}\right): \delta 162.3,157.3,136.5,129.7,128.9,126.9,126.6,123.9,63.1,35.9,32.7$, $29.5,29.0,26.0,25.6,18.3,-5.3$

ESI-HRMS (m/z): $[\mathrm{M}+\mathrm{H}]^{+}$calc'd for $\mathrm{C}_{22} \mathrm{H}_{35} \mathrm{~N}_{2} \mathrm{OSi}^{+}$: 371.2513 ; found: 371.2519 

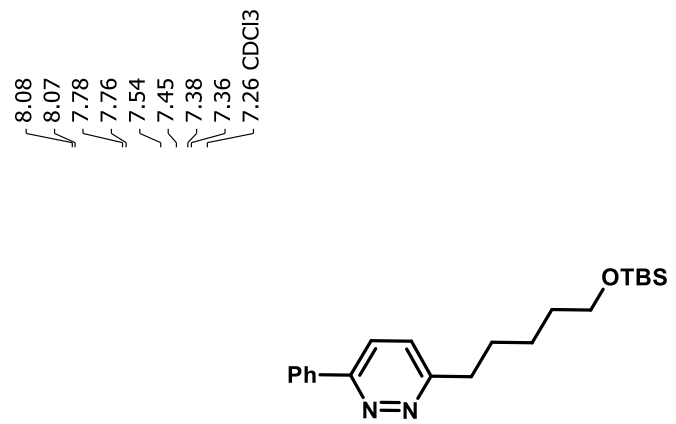

ํㅜㅇㅠํ 흉 웅

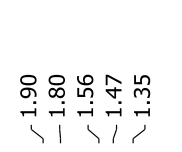

$\$$

12

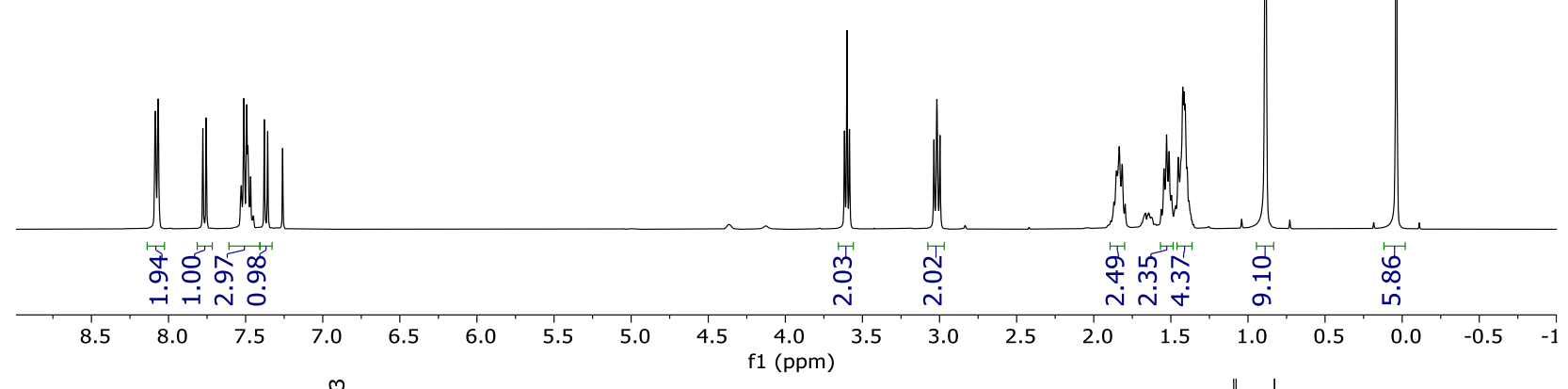

บั

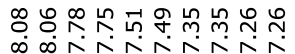

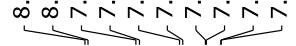

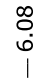

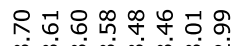

mimm min

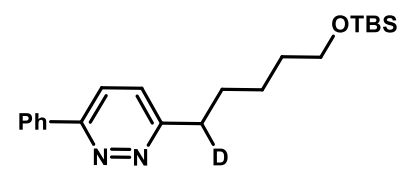

$12 \mathbf{a}$

Deuteration:

$(2.00-1.42) / 2.00=29 \%$

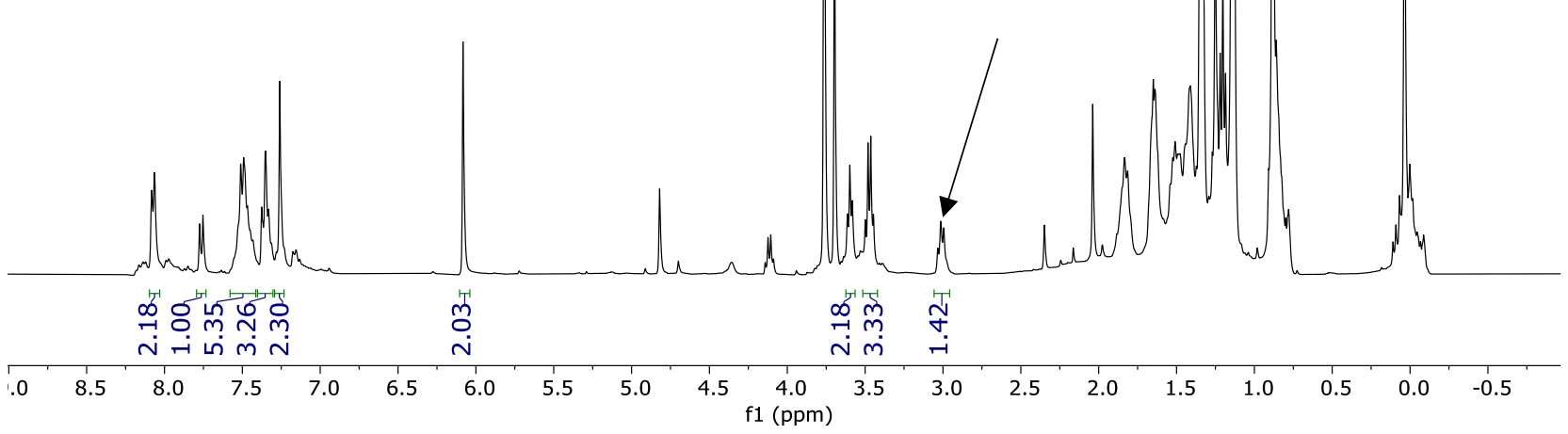


2-(butyl)-4,6-dimethoxy-1,3,5-triazine (13)

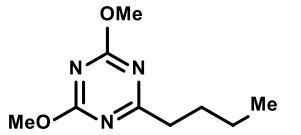

Compound 13 was prepared according to known procedure, ${ }^{2}$ and the characterization data match those previously reported in the literature.

${ }^{1} \mathbf{H}$ NMR $\left(400 \mathrm{MHz}, \mathrm{CDCl}_{3}\right): \delta 3.89(\mathrm{~s}, 6 \mathrm{H}), 2.59(\mathrm{t}, J=7.4 \mathrm{~Hz}, 2 \mathrm{H}), 1.63(\mathrm{dt}, J=13.1,7.6 \mathrm{~Hz}, 2 \mathrm{H}), 1.32$ $-1.19(\mathrm{~m}, 2 \mathrm{H}), 0.79(\mathrm{t}, J=7.4 \mathrm{~Hz}, 3 \mathrm{H})$

${ }^{13}$ C NMR (101 MHz, $\left.\mathrm{CDCl}_{3}\right): \delta 183.5,172.3,54.8,38.2,29.3,22.2,13.7$

ESI-HRMS (m/z): $[\mathrm{M}+\mathrm{H}]^{+}$calc'd for $\mathrm{C}_{9} \mathrm{H}_{16} \mathrm{~N}_{3} \mathrm{O}_{2}{ }^{+}$: 198.1237; found: 198.1243 


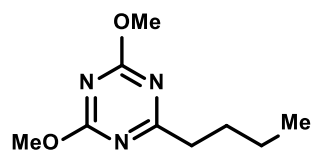

13

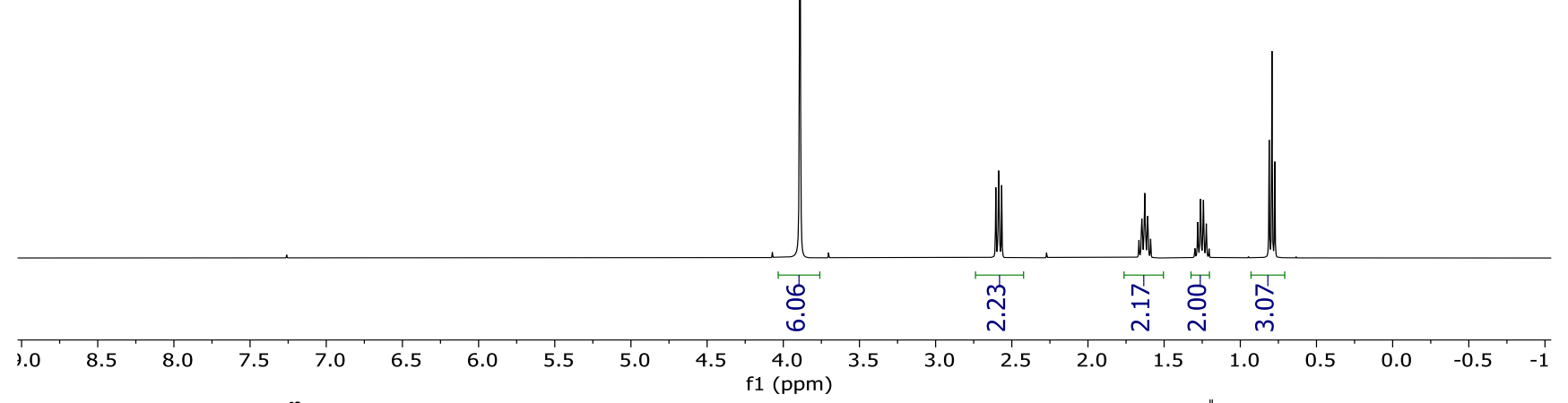

商

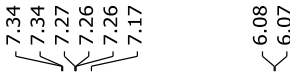

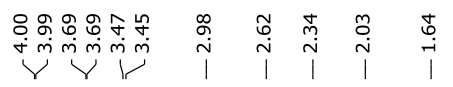

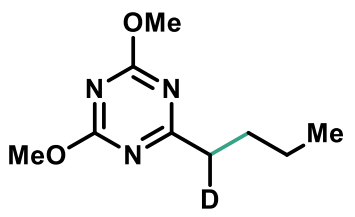

13a with significant degradation

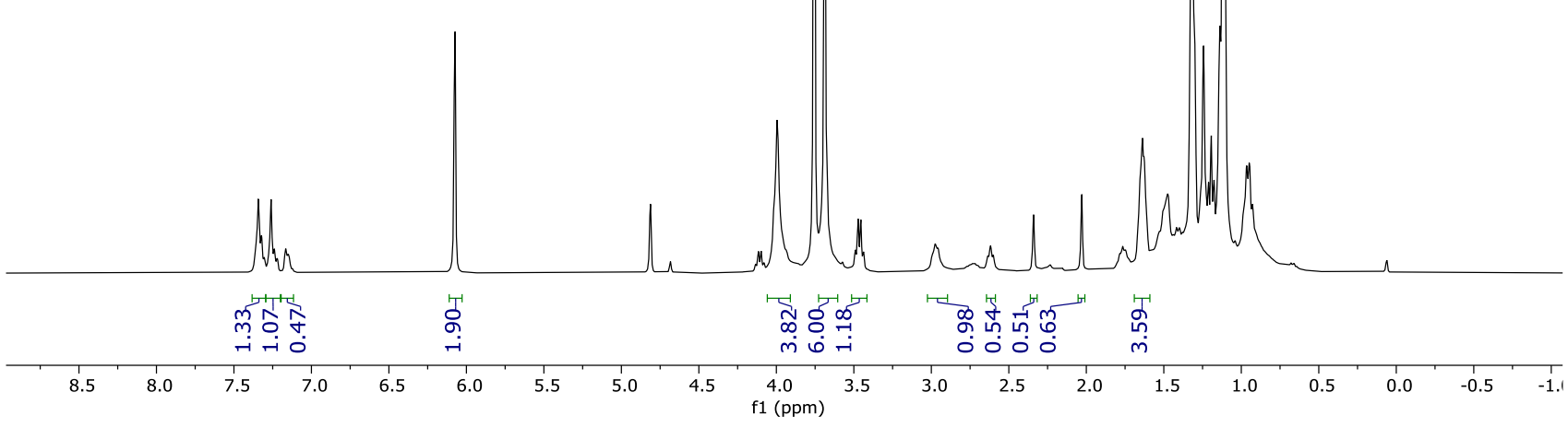




\section{General Experimental Procedure G for substrate preparation ${ }^{4}$}

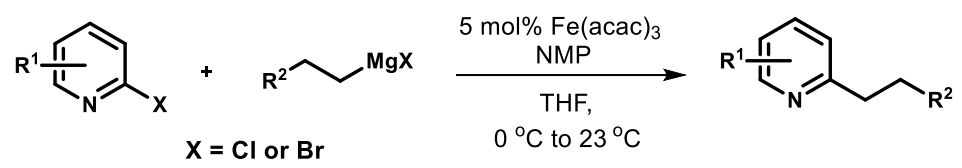

To a flame-dried microwave vial equipped with a magnetic stir bar was added magnesium (264 $\mathrm{mg}, 11 \mathrm{~mol}, 1.1$ equiv), a catalytic amount of $\mathrm{I}_{2}(5 \mathrm{mg})$, and THF (5 mL, 2.0 M). The resulting mixture was heated to $80{ }^{\circ} \mathrm{C}$, then removed the oil bath and alkyl bromide (10 mol, 1.0 equiv) was added via syringe dropwise over 10 minutes to maintain a gentle reflux. The reaction vessel was then stirred for 30 minutes at room temperature.

A flame-dried flask under $\mathrm{N}_{2}$ was charged with aryl halide (5.0 mmol, 1.0 equiv), $\mathrm{Fe}(\mathrm{acac})_{3}(88$ $\mathrm{mg}, 0.25 \mathrm{mmol} 5 \mathrm{~mol} \%)$, N-methylpyrrolidone $(3.0 \mathrm{~mL})$, and THF (30 mL, $0.15 \mathrm{M})$. A solution of alkyl magnesium bromide/chloride $(3.0 \mathrm{~mL}, 2.0 \mathrm{M}$ in THF, $6.00 \mathrm{mmol}, 1.2$ equiv) is added via syringe to the resulting red solution at $0{ }^{\circ} \mathrm{C}$, causing an immediate color change to dark brown or black. The resulting mixture was stirred at room temperature for 30 minutes, and the reaction was diluted with $\mathrm{Et}_{2} \mathrm{O}$ and is carefully quenched by the slow addition of aq. $1.0 \mathrm{M} \mathrm{HCl}(10 \mathrm{~mL})$. The reaction mixture was diluted with water $(20 \mathrm{~mL})$ and EtOAc $(20 \mathrm{~mL})$ and the organic phase was separated. The aqueous phase was extracted with EtOAc $(3 \times 25 \mathrm{~mL})$ and the combined organic extracts were washed with brine $(15 \mathrm{~mL})$, dried over anhydrous $\mathrm{Na}_{2} \mathrm{SO}_{4}$, filtered, and concentrated under reduced pressure by rotary evaporation. The crude mixture was purified by flash column chromatography on silica gel.

\section{2-methoxy-6-(3-phenylpropyl)pyridine (14)}

Compound 14 was prepared from 2-chloro-6-methoxypyridine and (3$\mathrm{MeO}_{\mathrm{Nh}}$ bromopropyl)benzene according to procedure $\mathrm{G}$.

${ }^{1} \mathbf{H}$ NMR $\left(400 \mathrm{MHz}, \mathrm{CDCl}_{3}\right) \delta 7.47(\mathrm{t}, J=8.1,7.7 \mathrm{~Hz}, 1 \mathrm{H}), 7.38-7.25(\mathrm{~m}, 2 \mathrm{H}), 7.25-7.11(\mathrm{~m}, 3 \mathrm{H})$, $6.71(\mathrm{~d}, J=7.2 \mathrm{~Hz}, 1 \mathrm{H}), 6.56(\mathrm{~d}, J=8.2 \mathrm{~Hz}, 1 \mathrm{H}), 3.94(\mathrm{~s}, 3 \mathrm{H}), 2.82-2.59(\mathrm{~m}, 4 \mathrm{H}), 2.22-1.87(\mathrm{~m}, 2 \mathrm{H})$.

${ }^{13}$ C NMR $\left(101 \mathrm{MHz}, \mathrm{CDCl}_{3}\right) \delta 166.4,160.2,142.4,139.7,131.4,126.9,115.7,108.7,77.8,77.5,77.2$, $53.6,37.7,35.9,30.3$.

GC-MS (m/z): $[\mathrm{M}+\mathrm{H}]^{+}$calc'd for $\mathrm{C}_{15} \mathrm{H}_{17} \mathrm{NO}^{+}: 227.1$; found: 227.1 

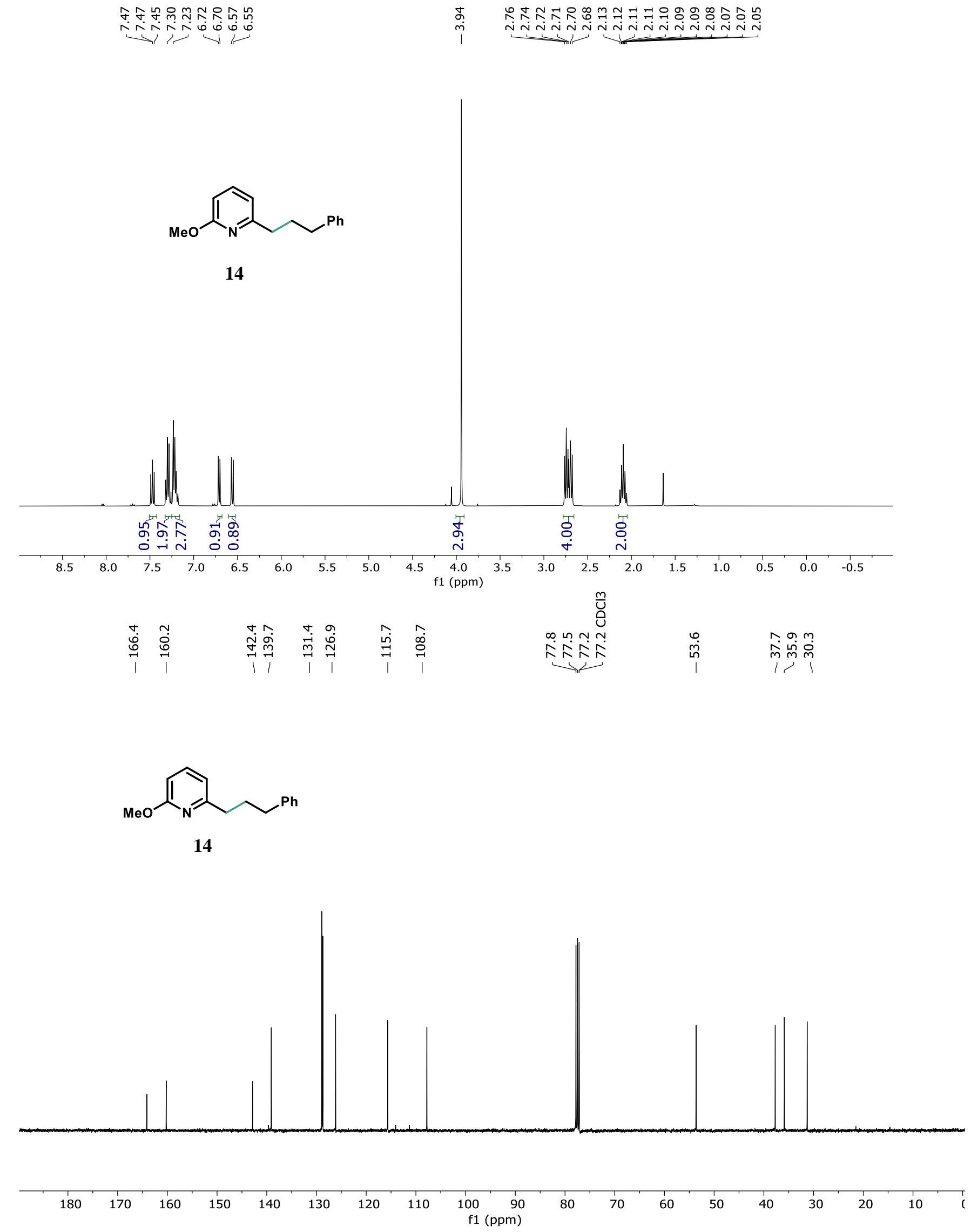


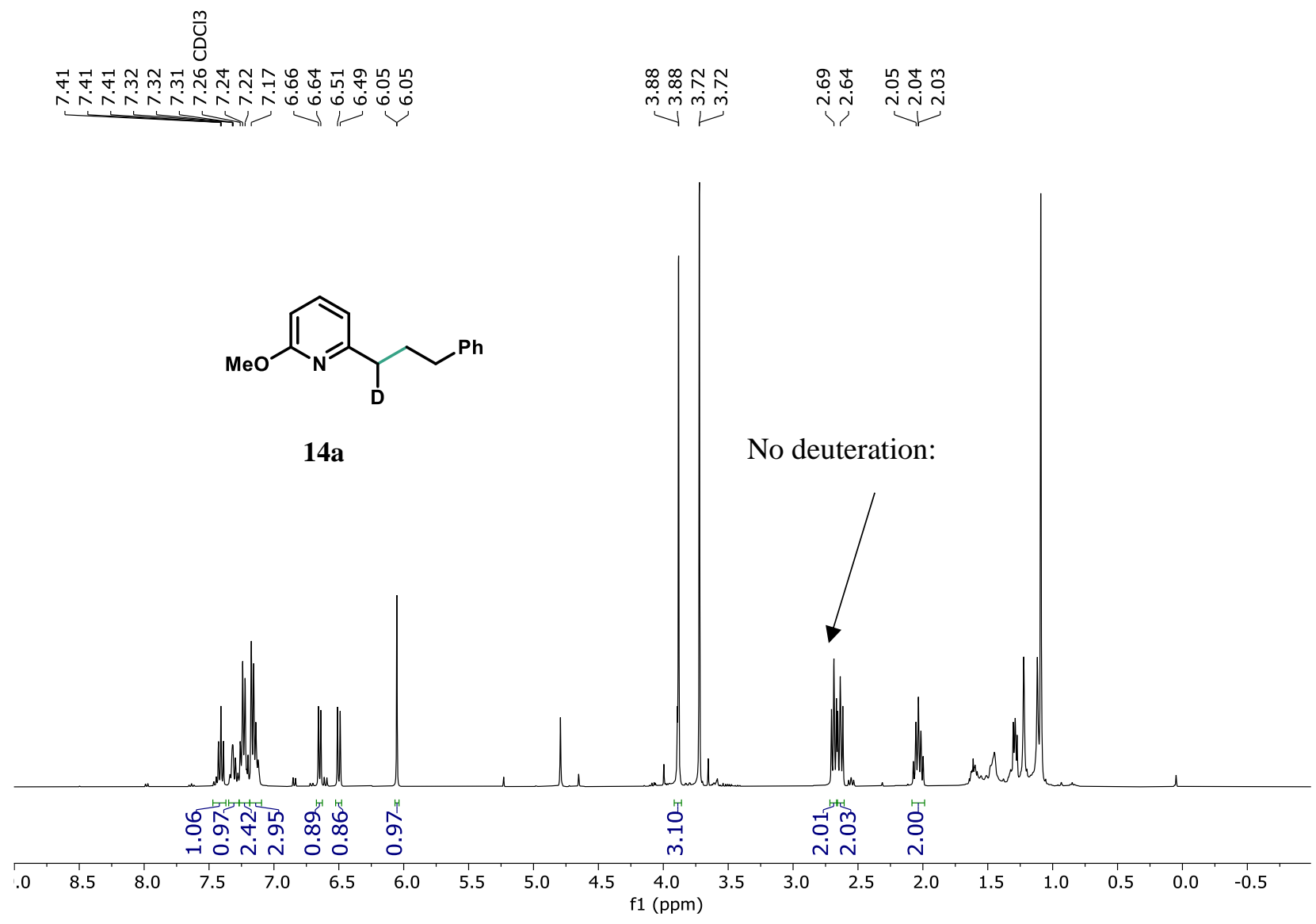




\section{4-(2-phenylethyl-1-d)-3-(trifluoromethyl)pyridine (15)}

$\mathrm{Ph}$ Compound $\mathbf{1 5}$ was prepared from 4-chloro-3-(trifluoromethyl)pyridine and (2-
bromoethyl)benzene according to procedure $\mathrm{G}$.

${ }^{1} \mathbf{H}$ NMR $\left(400 \mathrm{MHz}, \mathrm{CDCl}_{3}\right) \delta 8.84(\mathrm{~s}, 1 \mathrm{H}), 8.63(\mathrm{~d}, J=5.1 \mathrm{~Hz}, 1 \mathrm{H}), 7.35-7.26(\mathrm{~m}, 2 \mathrm{H}), 7.25-7.13$ (m, 4H), $3.16-3.04(\mathrm{~m}, 2 \mathrm{H}), 2.99-2.81(\mathrm{~m}, 2 \mathrm{H})$.

${ }^{13}$ C NMR $\left(101 \mathrm{MHz}, \mathrm{CDCl}_{3}\right) \delta 152.9,149.7(\mathrm{~d}, J=1.6 \mathrm{~Hz}), 147.3(\mathrm{q}, J=6.3 \mathrm{~Hz}), 140.4,128.8,128.6$, 126.7, 125.5, $125.0(\mathrm{q}, J=30.3 \mathrm{~Hz}), 124.3(\mathrm{q}, J=275.7 \mathrm{~Hz}), 36.9,34.4$.

GC-MS (m/z): $[\mathrm{M}+\mathrm{H}]^{+}$calc'd for $\mathrm{C}_{14} \mathrm{H}_{12} \mathrm{~F}_{3} \mathrm{~N}^{+}$: 251.1; found: 251.2 


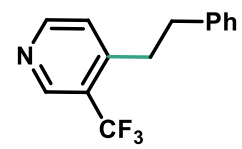

15

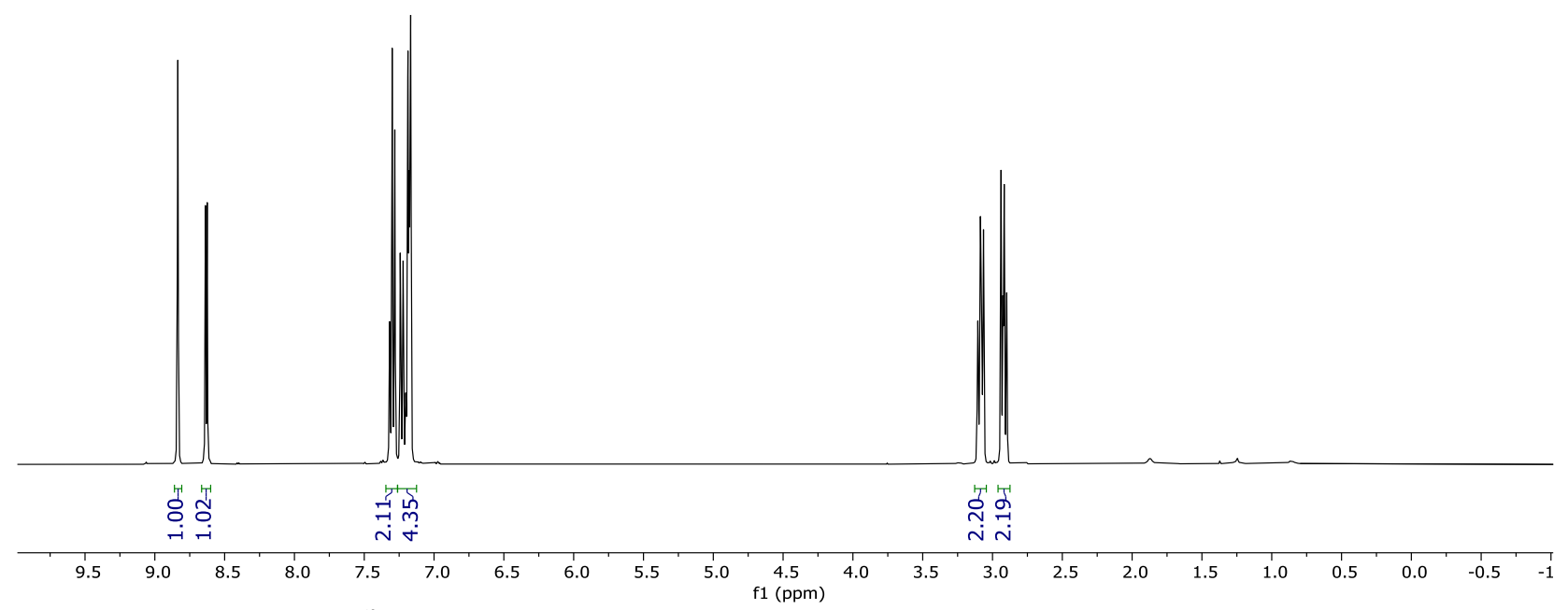

$\begin{array}{lll}0 & 0 \\ \infty & 0 \\ \infty & \infty & \infty \\ \infty & \infty\end{array}$

บับ

ำ

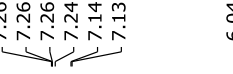

$\stackrel{\substack{+0}}{\substack{+\infty \\ \dot{\sim}}}$

시류ำ

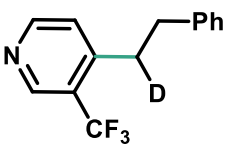

15a

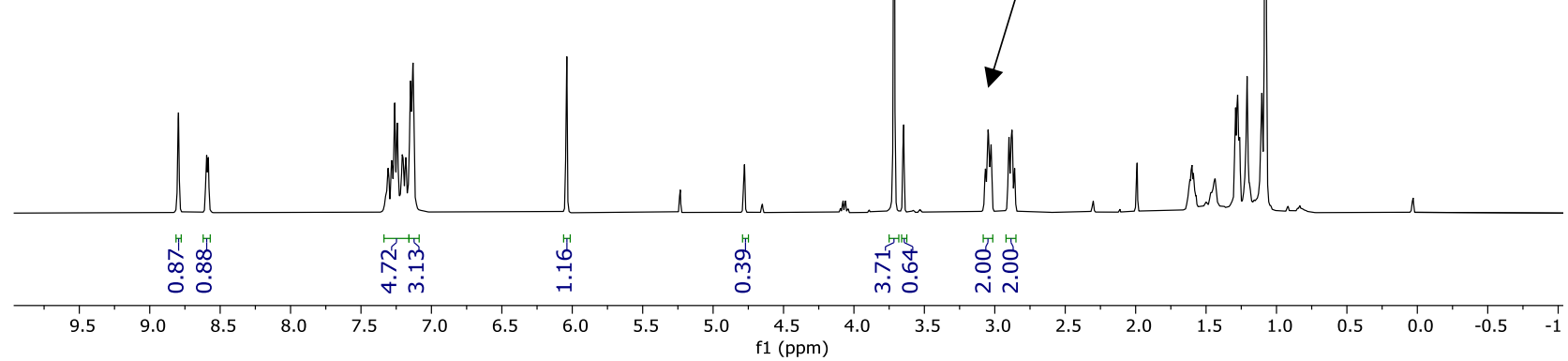



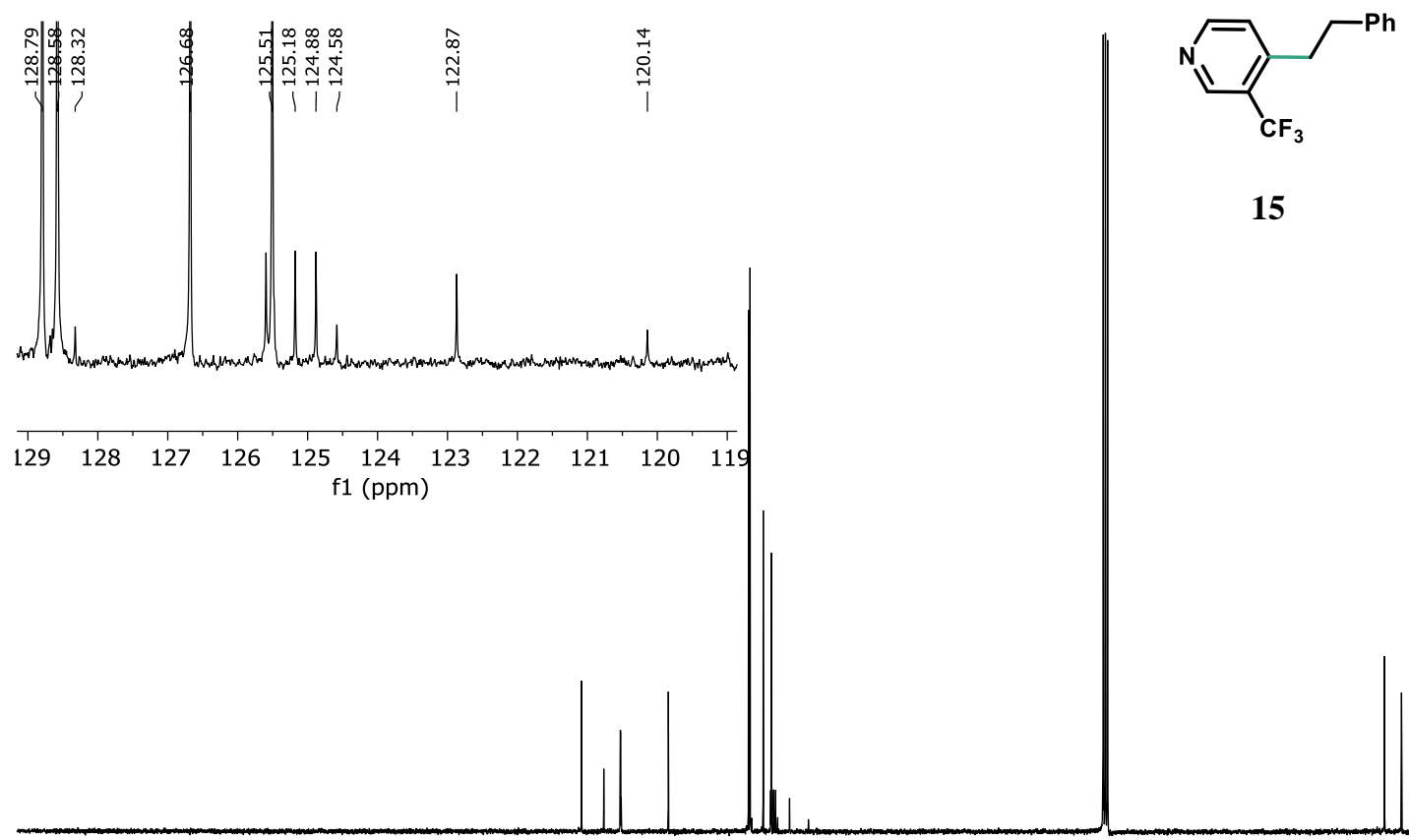

15

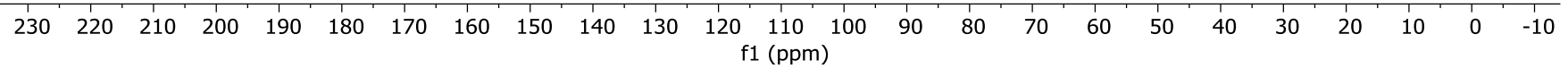


5,6,7,8-tetrahydroisoquinoline (16) (commercially available)

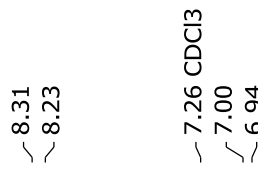

$\stackrel{i}{i} \quad \stackrel{r}{i}$

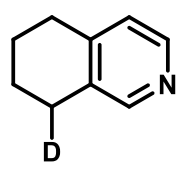

$16 a$

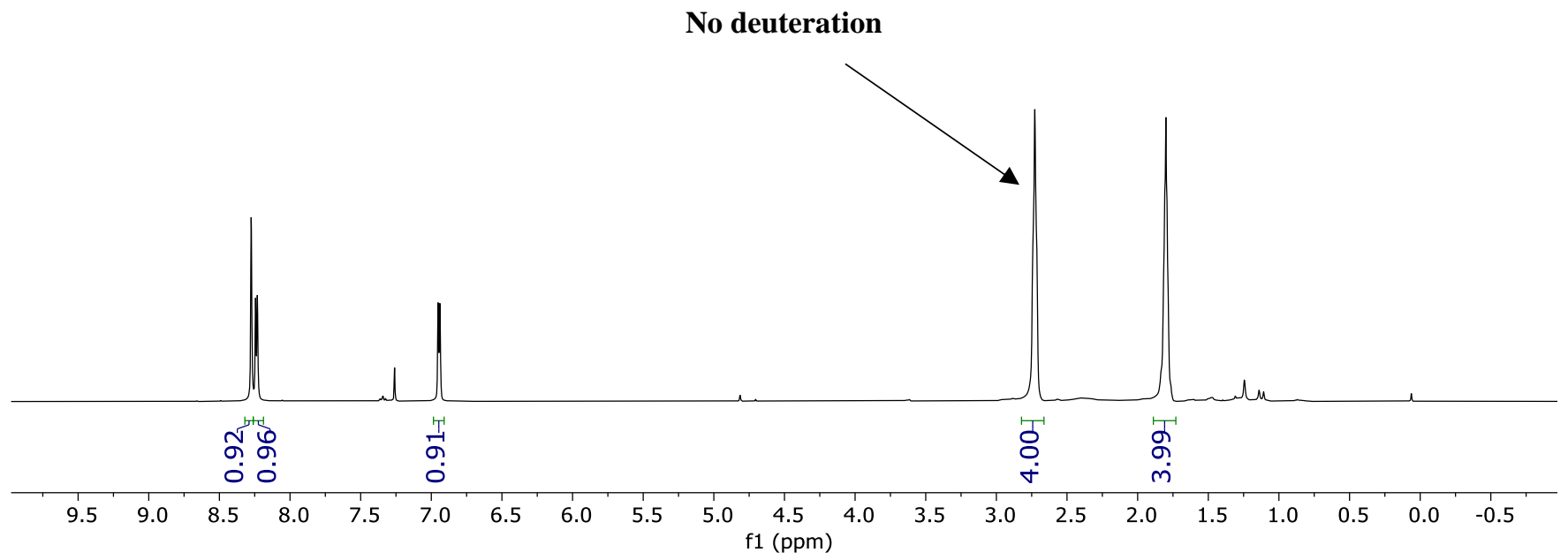




\section{$\underline{\text { References }}$}

1. Zhao, Y.; Chen, Y.; Newhouse, T. R. Allyl-Palladium Catalyzed $\alpha, \beta$-Dehydrogenation of Carboxylic Acids via Enediolates. Angew. Chem. Int. Ed. 2017, 56, 13122.

2. Zhang, P.; Huang, D.; Newhouse, T. R. Aryl-Nickel-Catalyzed Benzylic Dehydrogenation of Electron-Deficient Heteroarenes. J. Am. Chem. Soc. 2020, 142, 1757.

3. Mierde, H. V.; Van Der Voort, P.; De Vos, D.; Verpoort, F. A Ruthenium-Catalyzed Approach to the Friedländer Quinoline Synthesis. Eur. J. Org. Chem. 2008, 1625.

4. Fürstner, A.; Leitner, A.; Méndez, M.; Krause, H. Iron-Catalyzed Cross-Coupling Reactions. J. Am. Chem. Soc. 2002, 124, 13856. 